\title{
Restructuring Information Management in Building Design and Construction Using BIM as a Platform
}

\author{
Petrit Pasha $^{1, *}$, Mohd Tajuddin Bin Mohd Rasdi ${ }^{2}$ \\ ${ }^{1}$ Department of Urban Planning and Architectural Design, German University of Technology in Oman (GUtech), Sultanate of Oman \\ ${ }^{2}$ Faculty of Engineering, Technology and Built Environment, UCSI University, Malaysia
}

Received June 1, 2021; Revised January 18, 2022; Accepted February 8, 2022

Cite This Paper in the following Citation Styles

(a): [1] Petrit Pasha, Mohd Tajuddin Bin Mohd Rasdi, "Restructuring Information Management in Building Design and Construction Using BIM as a Platform," Civil Engineering and Architecture, Vol. 10, No. 2, pp. 600-619, 2022. DOI: 10.13189/cea.2022.100217.

(b): Petrit Pasha, Mohd Tajuddin Bin Mohd Rasdi (2022). Restructuring Information Management in Building Design and Construction Using BIM as a Platform. Civil Engineering and Architecture, 10(2), 600-619. DOI: 10.13189/cea.2022.100217.

Copyright $\odot 2022$ by authors, all rights reserved. Authors agree that this article remains permanently open access under the terms of the Creative Commons Attribution License 4.0 International License

\begin{abstract}
The purpose of this research is to restructure information management in the building design and construction process by using the BIM management platform. BIM has appeared in the scene of the architecture and construction chain by addressing multidisciplinary inefficiencies, incapabilities, disorganizations, and delays that have been rooted in the structure of architecture and building chain for over decades. However, the German architecture and construction chains are experiencing huge difficulties in accepting, endorsing, and understanding the BIM benefits, because there is no clear guidance and best practices in BIM implementation that comes as a reliable example. This research aims to present, explain, and analyze the differences between projects that have been designed and built in the traditional way and those which have utilized assets of the BIM management software. The investigation is based on two case studies - one project managed in the traditional non-BIM manner and the other one utilizing BIM as the main platform. The results reveal clearly that the BIM-involved project achieves higher accuracy, better design quality, effective team collaboration, and satisfying cost and time-efficiency compared to the traditional design and build project. Nevertheless, the results also reveal that the BIM software is underused or misused among the teams involved in the building chain.
\end{abstract}

Keywords Design Management, Construction, Cost Overrun, BIM

\section{Introduction}

There are two main approaches to the architectural design and the building process, the traditional one and the BIM approach.

The traditional and present ways of handling both design and building aspects are long due for a change as they create many problems, sometimes resulting in costs that exceed ten times the initial cost estimate.

Most of the monitored projects involving the traditional approach suffer from time inefficiency, engage large teams, and lack consistency among different phases of the design and building process. It is suspected that poor management in controlling information data may be the cause of unsatisfactory construction coordination and misunderstandings among different actors at the level of design, commissioning, and construction, and of the overall inadequate design and construction of the build environment.

Moreover, the relationship between the design and building construction chain actors is very loose, contrary to what the chain actors claim in the case study. Usually, these actors tend to keep to their territory of work and do not allow each other or other chain actors to cross their borders.

The lack of proper coordination and misunderstandings in managerial communication between the different design and building sections affect negatively the implementation of the projects in relation to the monetary costs, timeline, and operational efficiency. 
BIM data systems have appeared in the scene of the architecture and construction chain during the last decades, as a way to address multidisciplinary inefficiencies, incapability, disorganizations, and delays that have been rooted in the structure of the architectural and building chain over decades. The existing experience in managing design and construction projects suggests that BIM-involved projects are able to achieve higher accuracy, better design quality, increased team collaboration, and better cost and time-efficiency compared to traditional design and build projects. However, German architecture and construction chains are having huge difficulties in accepting, endorsing, and understanding the BIM Management Platform benefits, as there are no clear guidance and best practices in BIM implementation to be learned from, as a form of reliable examples.

At present, some data indicate a low use and misuse of the BIM software among the teams involved in the building chain, mainly due to the lack of information and knowledge. This creates problems in the development of the building and affects directly in terms of time and cost efficiency.

Findings and analysis from case studies reveal that if BIM data managerial platform is used more productively in the Design and Building Construction process, it will shorten the gaps between operators in the design and building construction process and automatically minimize the time-delays and costs of the projects.

\section{Research Gap - Literature Review}

According to the statistics, over the past two decades, social network analysis (SNA) has elicited increasing attention in construction project management (CPM). $\mathrm{BIM}$ is an excellent visualization tool based on a new concept that can improve the quality and efficiency of each link in the industry's chain of construction [21].

The central database of BIM also aids early-stage simulations as well as later-stage project documentation, due to the several means of collaboration tools it provides. However, there are also disadvantages to this approach, such as the learning curve of BIM tools and the difficulty of changing the traditional design process [22, 29].

One body of knowledge concerning BIM use in the construction industry relates to the management of projects concerning practice scheduling and lifecycle costs $[1,32,35]$. A larger body of concerns relates directly to the use of BIM in tracking architectural projects in conservation, typological buildings such as high rises, and evaluation of success rates in predicting construction outcomes in practice [2-5, 31,34]. Another popular BIM usage is the aspect of safety measures against accidents in construction projects $[6,7,30]$. A growing concern among computer software analysts is the development of the BIM software packages and their evaluation in real practice $[8-10,33,35,36]$. The massive use of BIM as a performance tool is related directly to after-completion project analysis of building construction [11-14].

Coming from an academic perspective, most of the researchers do not have a direct practice experience of a full cycle of design and building process. Most of them conduct their analysis after the design is finished or once the building is constructed. When observing the project from the outside, one can visualize the final product, but may not fully understand and foresee the g problems that may occur during the process, Therefore, most of the researchers have not developed clear ideas and visions about the fundamentals of the BIM platform.

The lack of a full understanding of issues creates a huge gap between academic research industry manufacturing and architectural design and construction management.

On the other side, there is a lack of a ready pool of skilled workers who are able to develop BIM into the construction industry, specifically in the design phase to achieve its best practices [19-20].

From the researcher's experience, this is directly affecting the building industry, by creating a divergence on the strategic development of the BIM platform and by creating countless cracks in the design and building process between the different stakeholders and researchers.

As of now, one can easily detect the big influence from the industrial sector on BIM platforms - software developers by monopolizing the possibilities as per the request of Industrial manufacturing, and leaving architects with no possibilities on parametrizing elements, sizes, shapes, and structures.

Even though most of the BIM software platforms have developed the Integrated Project Delivery (IPD) and other frameworks to provide a solution to reduce the risk of overspending during project construction, as well as to predict the cost of a life-cycle of activity, there is still a very big division between the design and building construction chain $[23-28,35]$. This division on the other side affects directly the researchers by having no access to BIM platform development. It makes every section of the building construction chain work within its pyramid.

\section{Objective and Methodology}

To understand the gap in the existing literature, the researchers have reviewed and analyzed over 50 published research papers from Scopus index journals on the topic of BIM and Design Management areas. Furthermore, the researchers have compared two cases study projects using traditional and BIM methods. The research presents data from two real design and building cases and this is important as it will fill in the gap that exists in the literature. The comparison between the two case study projects provides significant information on the features that would need to be fixed and updated using the BIM 
design and building process.

The main objective of this research is to identify efficient ways in which the BIM platform is utilized and can produce better time management and quality interaction between the professionals involved in the design and building process. This will be done through discovering the advantages, benefits, assets, differences, and possibilities from the two projects designed and built by architectural and engineering offices in Germany where one of the researchers has worked as a project leader/consultant.

The scope of this research is limited to German architectural practices and the information available within the legal boundary of professional practice in the country.

The two case study projects are analyzed, structured, and presented by the researchers based on a book of fees and time-schedule systems of German Architecture and Engineering, called HOAI. HOAI is a book, which includes most of the architecture and engineering regulations based on Federal German Government Laws. The book describes the entire process and procedures from the design phases to the completion of the construction phases.

The process of analysis involves two tables based on the German structure of the design and construction development:

- Table 1 presents the development of the projects through all phases of design and construction based on a schedule.
- Table 2 presents the automatic calculation of the days and costs related to the projects' phases described in Table 1.

The two case studies are described through these tables, representing two different ways of architecture management development:

Case study 1: Traditional Architecture Management

Case study 2: BIM Architecture Management

To explain the whole process, the researcher has structured the analysis into different phases of project development, based on the German architecture and engineering project phases system:

1. Pre-Phases,

2. Architectural Design, Supervision Phases,

3. Revision Phases,

4. Building Construction Phases,

5. Expected Timeline Schedule - Total.

In the table below (Table 1), are listed the two case study projects, analyzed within the phases of the German Architectural Design and Construction Management.

The cases studies presented in Table 1 are listed in the following order:

1. Case Study 1 (Traditional Project/ non-BIM project)

2. Case Study 1 (If the project will be designed on BIM)

3. Case Study 2 (BIM Project)

4. Case Study 2 (If the project will be designed on BIM at $100 \%)$ 
Table 1. Example of the Table Comparison Structure for the Case Study of Traditional Management and BIM Project

\begin{tabular}{|c|c|c|c|c|c|c|c|c|c|c|c|c|c|c|c|c|c|c|c|}
\hline $\begin{array}{l}\text { Project / Project } \\
\text { Section - DAY }\end{array}$ & 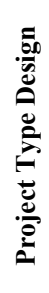 & 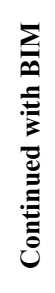 & 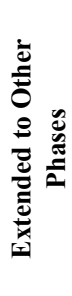 & 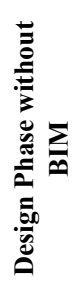 & 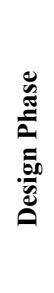 & 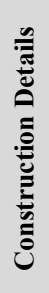 & 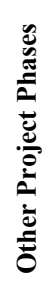 & 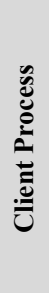 & 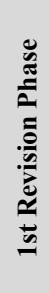 & 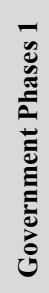 & 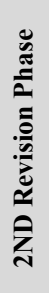 & 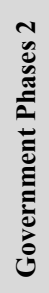 & 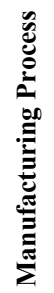 & 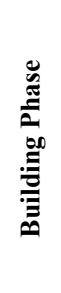 & 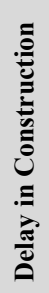 & $\begin{array}{l}0 \\
\vdots \\
0 \\
0 \\
0 \\
0 \\
0\end{array}$ & 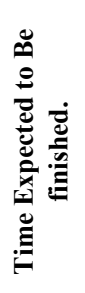 & 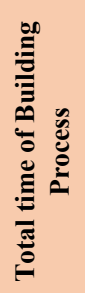 & 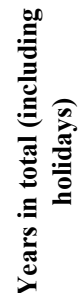 \\
\hline $\begin{array}{c}\text { P. W. School } \\
\text { (traditional/non-BIM } \\
\text { project) }\end{array}$ & & & & & & & & & & & & & & & & & & & \\
\hline $\begin{array}{l}\text { P.W. School (if it is a } \\
\text { BIM project) }\end{array}$ & & & & & & & & & & & & & & & & & & & \\
\hline $\begin{array}{l}\text { ALN Office and } \\
\text { Residential (BIM } \\
\text { project) }\end{array}$ & & & & & & & & & & & & & & & & & & & \\
\hline $\begin{array}{c}\text { ALN Office and } \\
\text { Residential } \\
\text { (BIM project } 100 \% \text { ) }\end{array}$ & & & & & & & & & & & & & & & & & & & \\
\hline
\end{tabular}


Table 2. Hazard Colour Key / Project Efficiency Level for Table Comparison Structure for the Study 1 and 2

\begin{tabular}{|c|c|c|c|}
\hline $\mathbf{N r}$ & $\begin{array}{c}\text { PROJECT EFFICIENCY } \\
\text { LEVEL }\end{array}$ & $\begin{array}{c}\text { COLOR } \\
\text { INDICATION }\end{array}$ & DAYS \\
\hline 1 & HIGH EFFICIENCY & 0 & DAYS \\
\hline 2 & GOOD EFFICIENCY & 10 & DAYS \\
\hline 3 & MMEDIUMEFFICIENCY & 20 & DAYS \\
\hline 4 & LOW EFFICIENCY & 30 & DAYS \\
\hline 5 & VERY LOW EFFICIENCY & 40 & DAYS \\
\hline 6 & HAZARD SITUATION & 50 & DAYS \\
\hline
\end{tabular}

The color range code from green (very high efficiency) to orange (optimal efficiency) to red (very low efficiency) represents the project qualities within BIM and Traditional Project Management (Table 2).

\section{Findings of Research}

The analysis of the BIM management data in design and construction is made through two different case study projects. The approach in this section is about discovering the advantages, benefits, assets, differences, and possibilities from two Traditional and BIM design and build projects by architecture and engineering offices where one of the researchers worked as a project leader/consultant based in Germany.

\subsection{Case Study One: P. W. School}

ALN together with B. Architekten developed and designed the P. W. School project in the classic way (Table $3)$. The space program was developed and designed in a $2 \mathrm{D}$ CAD plan and a volume model in Rhino. The project has been suspended for over a year because of high-cost estimation. Currently, (2021) the project has resumed its building process.

The reason why the researchers decided to analyze this project is that it involved two architecture offices that dealt with Large Scale Projects, public investment, different software programs (2D and $3 \mathrm{D})$ as well as terrain difficulties. This project involves several 2D sections build up in traditional CAD software, representing the exact difficult landscape of the case.

Table 3. Case Study One, Determining the chronology of the design phases at the P.W. School (Author Source)

\begin{tabular}{|c|c|c|c|c|c|c|c|c|c|c|c|c|c|c|c|c|c|c|c|c|c|}
\hline \multicolumn{22}{|c|}{ Case study 1 -: P.W. School } \\
\hline \multirow{2}{*}{ Name } & \multicolumn{4}{|c|}{ 1st Year } & \multicolumn{4}{|c|}{ 2nd Year } & \multicolumn{5}{|c|}{ 3rd Year } & \multicolumn{4}{|c|}{ 4th Year } & \multicolumn{3}{|c|}{ 5th Year } & \multirow{2}{*}{$\frac{6 \text { th Year }}{1}$} \\
\hline & 1 & 2 & 3 & 4 & 1 & 2 & 3 & 4 & 1 & 2 & 3 & 4 & 1 & 2 & 3 & 4 & 1 & 2 & 3 & 4 & \\
\hline Basic Determination & & & & & & & & & & & & & & & & & & & & & \\
\hline $\begin{array}{l}\text { Development of the } \\
\text { pedagogical concept }\end{array}$ & & & & & & & & & & & & & & & & & & & & & \\
\hline $\begin{array}{l}\text { Creation of a rough site } \\
\text { plan, first floor plans, } \\
\text { section, and views }\end{array}$ & & & & & & & & & & & & & & & & & & & & & \\
\hline Basic Determination & & & & & & & & & & & & & & & & & & & & & \\
\hline $\begin{array}{l}\text { Distribution of function } \\
\text { on the property and in } \\
\text { the building }\end{array}$ & & & & & & & & & & & & & & & & & & & & & \\
\hline $\begin{array}{l}\text { Configuration of the } \\
\text { building }\end{array}$ & & & & & & & & & & & & & & & & & & & & & \\
\hline Pre-Planning & & & & & & & & & & & & & & & & & & & & & \\
\hline $\begin{array}{l}\text { Development of the } \\
\text { ground plans }\end{array}$ & & & & & & & & & & & & & & & & & & & & & \\
\hline Sections and views & & & & & & & & & & & & & & & & & & & & & \\
\hline $\begin{array}{l}\text { Creation of the open } \\
\text { space planning }\end{array}$ & & & & & & & & & & & & & & & & & & & & & \\
\hline Conceptual design & & & & & & & & & & & & & & & & & & & & & \\
\hline further processing & & & & & & & & & & & & & & & & & & & & & \\
\hline $\begin{array}{l}\text { Determination of all } \\
\text { surfaces }\end{array}$ & & & & & & & & & & & & & & & & & & & & & \\
\hline $\begin{array}{c}\text { Planning of heating and } \\
\text { ventilation }\end{array}$ & & & & & & & & & & & & & & & & & & & & & \\
\hline $\begin{array}{c}\text { Planning of open space, } \\
\text { parking } \\
\end{array}$ & & & & & & & & & & & & & & & & & & & & & \\
\hline $\begin{array}{l}\text { Preparation of the cost } \\
\text { calculation }\end{array}$ & & & & & & & & & & & & & & & & & & & & & \\
\hline
\end{tabular}


Table 3 Continued

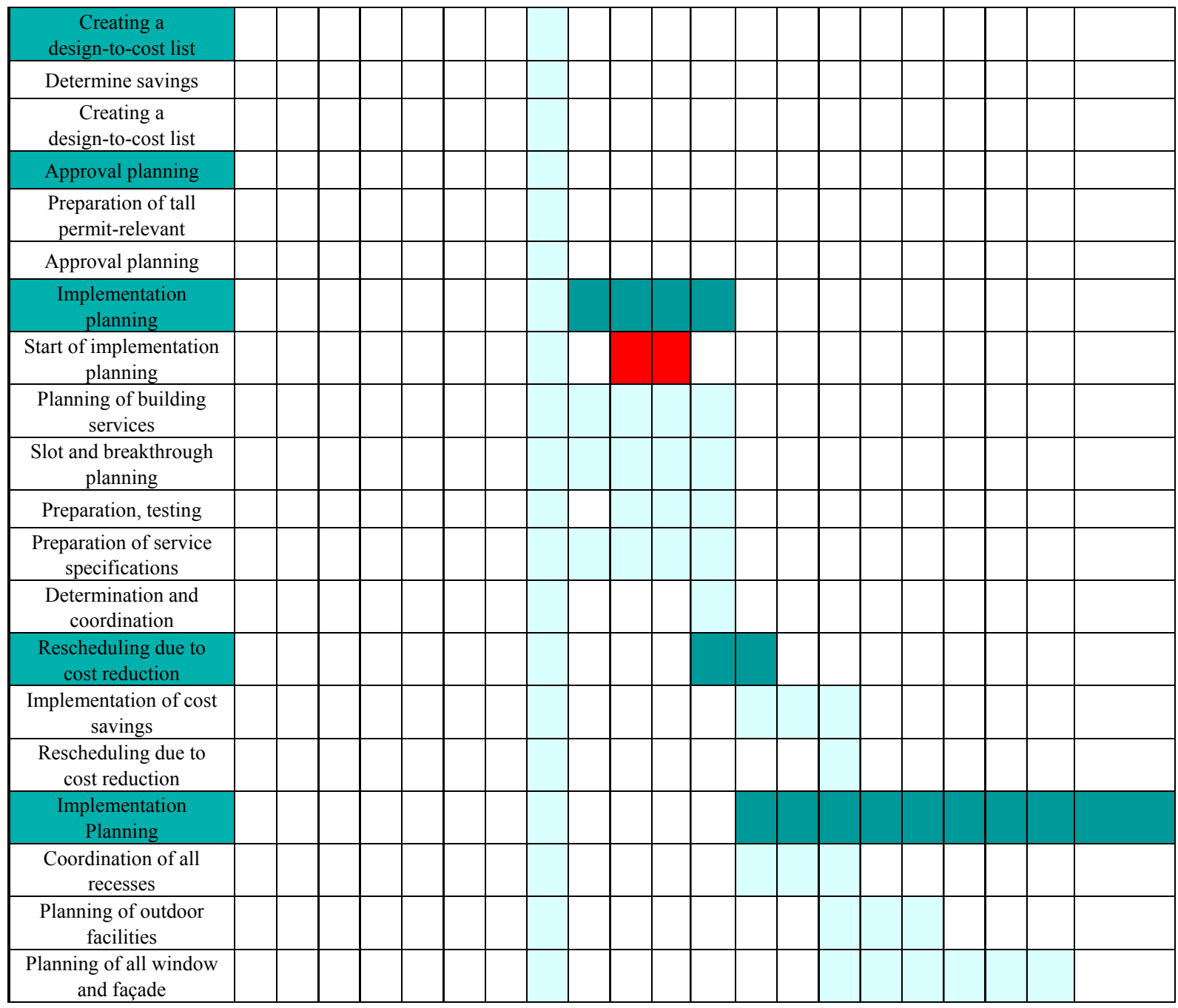

\subsection{Case Study Two - ALN Office, Residential Housing}

The architectural office ALN, was built after a three-year planning and construction period of a new residential and commercial building placed directly at the castle hill, on the edge of the historic Gothic town center of Landshut (Table 4). ALN was able to organize and drive the design process faster and more efficiently. Due to the different geometry and topography alone, a 3D model of the building's cubature together with the terrain was essential. The researcher and others could integrate the models created in ArchiCAD directly with Rhino and
Grasshopper. In this way, they had better control over the building volume in all phases, especially given the building's slightly different geometry, which doesn't utilize ordinary shape solutions where each wall is at right angles. This Project was the pioneer of 3D BIM thinking in the ALN office. After this project, ALN has shifted all architecture and engineering offices into the BIM system.

By making a comparison between Table $3 \& 4$, one can visualize the hazard indications on the traditional project structures, which are always related to design, building, and delays schedule. 
Table 4. Case Study Two, Determining the chronology of the design phases at the ALN Office, Residential Housing (Author Source)

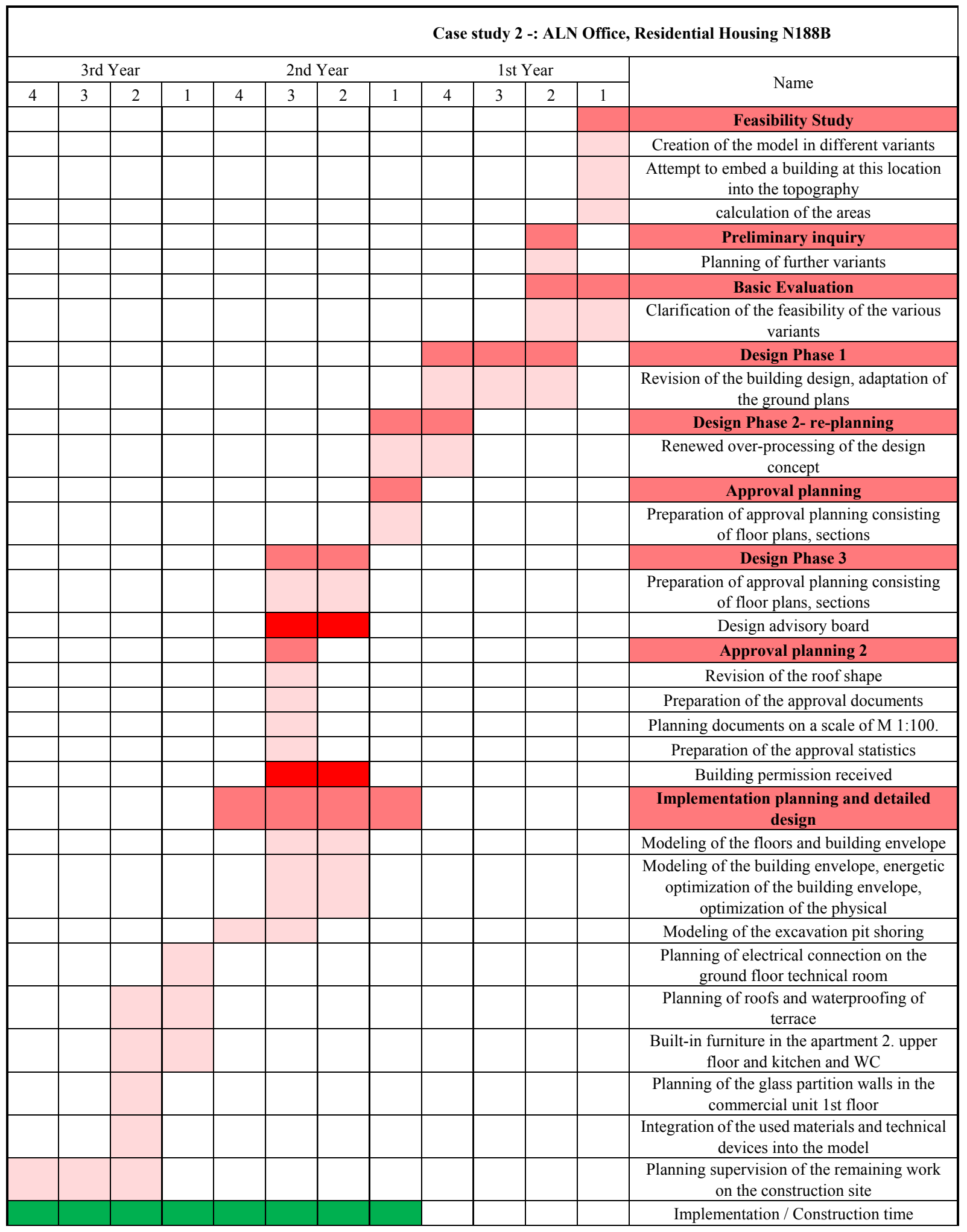

In the tables below (Table 5a and $5 \mathrm{~b}$ ), the authors of the paper have listed two of the projects which are case studies 1 and 2. Both cases are analyzed within the phases of the Architectural Design Management, represented on WORKING DAYS EFFICIENCY (Table 5a) and

\section{COST ESTIMATION EFFICIENCY (Table $5 b$ ).}

The graphic chart is based on all phases of design and building construction, including the most important phases of the design and building process.

The color range code from green (very high efficiency) 
to orange (optimal efficiency) the red (very low efficiency) represents the project qualities within BIM and Traditional Project Management.

Tables 5a and 5b represent the total process of Design, Revisions, and Construction Phase for the Case Studies:

1. Case Study 1 (Traditional Project);

2. Case Study 1 (If the project will be designed on BIM);

3. Case Study 2 (BIM Project);

4. Case Study 2 (If the project will be designed on BIM at $100 \%)$.

Within comparing these graphic charts analyses, one can also detect that the price and the time are significantly improved within the BIM management system, while the traditional planning and designing takes much more time, involves numerous delays, and most importantly, leads to an economically inefficient project.

When comparing the traditional system of planning and BIM planning, it is important to analyze and discuss the main phases of the project development: design, manufacturing, and building construction phase. These phases play a very significant role in the building chain.

According to that, the authors of the paper have divided the schedule of the project for Case Study 1 and Case Study 2 into the Design Phase and Delay / Revisions Phase. Design Phase is represented in Tables 6a and 6b, Delay / Revisions Phase is represented in Tables $7 \mathrm{a}$ and $7 \mathrm{~b}$.

Table 5a. Comparison of Case Study 1 -Traditional Management and Case Study 2 - (Total Process) - BIM Project Working Days Efficiency (Author Source)

\begin{tabular}{|c|c|c|c|}
\hline $\mathbf{N r}$ & PROJECT EFFICIENCY LEVEL & COLOR INDICATION & DAYS \\
\hline 1 & HIGH EFFICIENCY & 0 & DAYS \\
\hline 2 & GOOD EFFICIENCY & 10 & DAYS \\
\hline 3 & MMEDIUMEFFICIENCY & 20 & DAYS \\
\hline 4 & LOW EFFICIENCY & 30 & DAYS \\
\hline 5 & VERY LOW EFFICIENCY & 40 & DAYS \\
\hline 6 & HAZARD SITUATION & 50 & DAYS \\
\hline
\end{tabular}

Table 5b. Design, Manufacturing and Building Phases for the CASE study 1 and 2 (Total Process) - Cost Efficiency (Author Source)

\begin{tabular}{|c|c|c|c|c|c|c|c|c|c|c|c|c|c|c|c|c|c|c|c|}
\hline 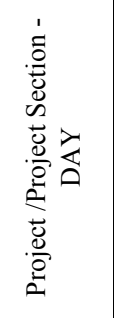 & 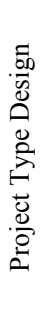 & 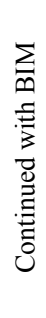 & 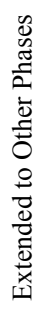 & 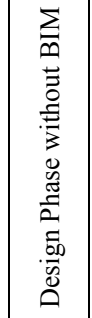 & 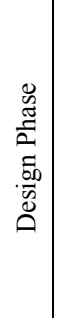 & 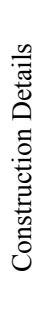 & 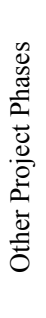 & 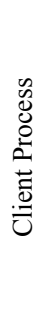 & 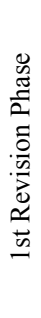 & 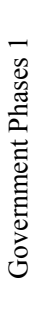 & 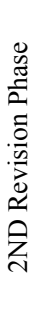 & 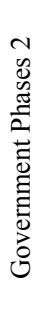 & 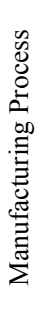 & 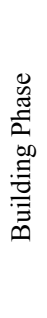 & 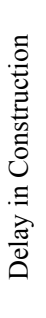 & 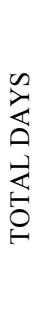 & 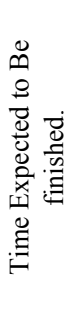 & 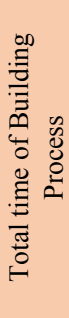 & 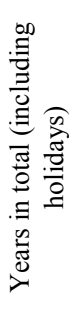 \\
\hline 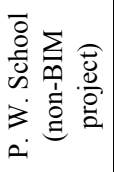 & 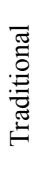 & 胥 & 蔦 & 0 & ஓ্ল & $\infty$ & \& & $\underset{\mathrm{N}}{\mathbf{N}}$ & లి & ஓ & $\cong$ & $\cong$ & 8 & ঙิ & q & $\begin{array}{l}\stackrel{0}{ } \\
\end{array}$ & $\stackrel{\infty}{\stackrel{\infty}{+}}$ & $\begin{array}{l}8 \\
\infty\end{array}$ & 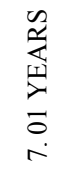 \\
\hline 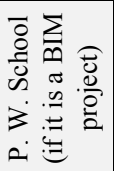 & $\sum_{m}$ & $\sum_{\underline{m}}$ & $\sum_{\infty}$ & 0 & $\stackrel{\wp}{7}$ & 0 & 0 & సి & $\cong$ & तి & in & $n$ & $\approx$ & \& & ి & $\stackrel{8}{\circ}$ & 0 & \&ి & 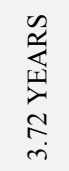 \\
\hline 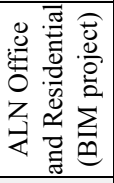 & 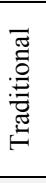 & $\sum_{m}$ & $\sum_{\infty}$ & 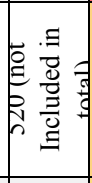 & \& & $\approx$ & 0 & $\stackrel{\varrho}{=}$ & 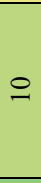 & లి & in & 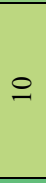 & ஜ & $\underset{\sim}{\stackrel{0}{*}}$ & $\cong$ & 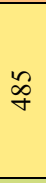 & 0 & $\stackrel{\substack{\infty \\
+}}{ }$ & 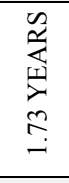 \\
\hline 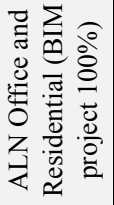 & $\sum_{\bar{m}}$ & $\sum_{\bar{m}}$ & 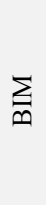 & 0 & $\infty$ & 0 & 0 & 0 & $n$ & $n$ & 0 & 0 & $\stackrel{ }{=}$ & $\stackrel{\text { P }}{\sim}$ & 0 & 离 & 0 & 市 & 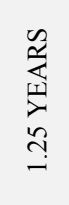 \\
\hline
\end{tabular}


Table 5b. Continued

\begin{tabular}{|c|c|c|c|c|c|c|c|c|c|c|c|c|c|c|c|c|c|c|c|}
\hline 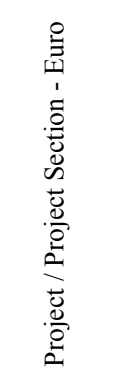 & 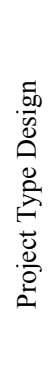 & 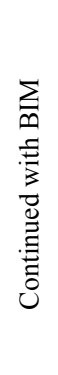 & 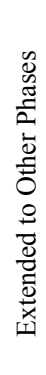 & 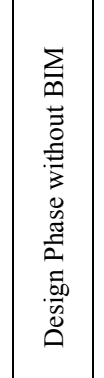 & 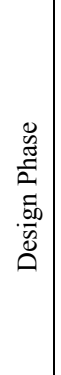 & 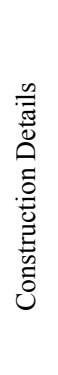 & 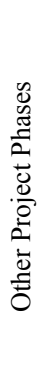 & 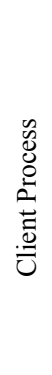 & 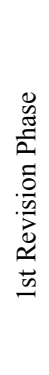 & 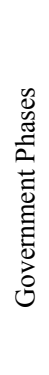 & 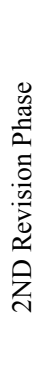 & 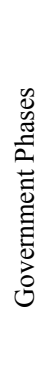 & 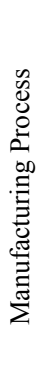 & 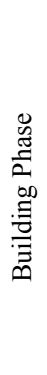 & 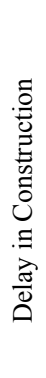 & 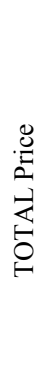 & 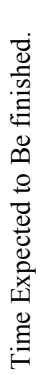 & 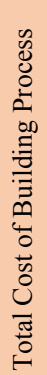 & 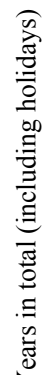 \\
\hline 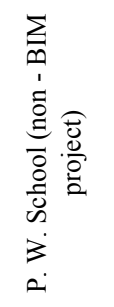 & 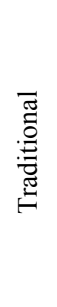 & 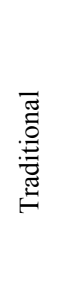 & 胥 & 0 & 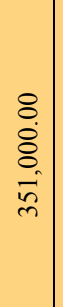 & $\begin{array}{l}8 \\
\dot{8} \\
8 \\
8 \\
8 \\
8\end{array}$ & $\begin{array}{l}8 \\
8 \\
8 \\
8 \\
0\end{array}$ & $\begin{array}{l}8 \\
8 \\
8 \\
\infty \\
\text { î } \\
\infty \\
-1\end{array}$ & $\begin{array}{l}8 \\
8 \\
8 \\
n \\
\text { I } \\
=\end{array}$ & $\begin{array}{l}8 \\
8 \\
8 \\
8 \\
8\end{array}$ & $\begin{array}{l}8 \\
0 \\
0 \\
i n \\
0 \\
n\end{array}$ & $\begin{array}{l}8 \\
8 \\
8 \\
8 \\
i\end{array}$ & 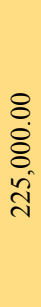 & $\begin{array}{l}8 \\
8 \\
8 \\
n \\
n \\
n\end{array}$ & 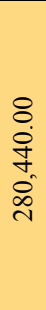 & $\begin{array}{l}8 \\
0 \\
0 \\
0 \\
\infty \\
0 \\
\text { n. }\end{array}$ & $\begin{array}{l}8 \\
8 \\
8 \\
8 \\
8 \\
0\end{array}$ & $\begin{array}{l}8 \\
0 \\
0 \\
0 \\
\infty \\
8 \\
0 \\
0\end{array}$ & 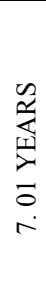 \\
\hline 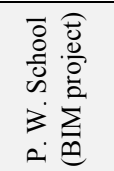 & $\sum_{m}$ & $\sum_{\emptyset}$ & $\sum_{m}$ & 0 & 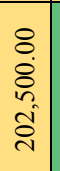 & $\stackrel{8}{8}$ & $\stackrel{8}{\circ}$ & $\begin{array}{l}8 \\
0 \\
0 \\
n \\
0\end{array}$ & 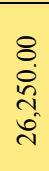 & $\begin{array}{l}8 \\
8 \\
8 \\
8 \\
\text { i. }\end{array}$ & $\begin{array}{l}8 \\
8 \\
i \\
n \\
\infty\end{array}$ & $\begin{array}{l}8 \\
\dot{8} \\
8 \\
0\end{array}$ & $\begin{array}{l}8 \\
\stackrel{8}{0} \\
\stackrel{n}{n} \\
\dot{f}\end{array}$ & $\begin{array}{l}8 \\
8 \\
8 \\
8 \\
8\end{array}$ & $\begin{array}{l}8 \\
8 \\
0 \\
i \\
6\end{array}$ & 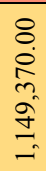 & $\stackrel{8}{\circ}$ & 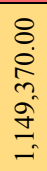 & 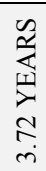 \\
\hline 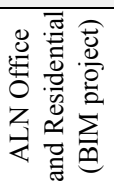 & 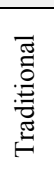 & $\sum_{\infty}$ & $\sum$ & 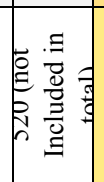 & $\begin{array}{l}8 \\
\dot{8} \\
8 \\
\dot{0} \\
0\end{array}$ & $\begin{array}{l}8 \\
\dot{0} \\
i \\
6 \\
0\end{array}$ & $\stackrel{8}{0}$ & $\begin{array}{l}8 \\
0 \\
n \\
n \\
\infty\end{array}$ & $\begin{array}{l}8 \\
8 \\
8 \\
0 \\
0\end{array}$ & $\begin{array}{l}8 \\
0 \\
6 \\
6 \\
n \\
n\end{array}$ & $\begin{array}{l}8 \\
\dot{8} \\
8 \\
\text { m. }\end{array}$ & $\begin{array}{l}8 \\
8 \\
0 \\
n \\
\infty\end{array}$ & $\begin{array}{l}8 \\
8 \\
8 \\
8 \\
0 \\
\text { in }\end{array}$ & $\begin{array}{l}8 \\
8 \\
8 \\
8 \\
0\end{array}$ & 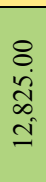 & $\begin{array}{l}8 \\
\text { ปิ } \\
\infty \\
\text { ปิ }\end{array}$ & $\stackrel{8}{\circ}$ & $\begin{array}{l}8 \\
\varkappa \\
\infty \\
\infty \\
\text { ปे }\end{array}$ & 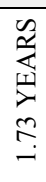 \\
\hline 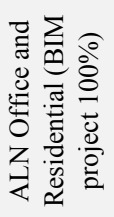 & $\sum_{\infty}$ & $\sum_{\infty}$ & $\sum_{m}$ & 0 & $\begin{array}{l}8 \\
\dot{8} \\
8 \\
\dot{+}\end{array}$ & $\stackrel{8}{0}$ & $\begin{array}{l}8 \\
0\end{array}$ & 8 & 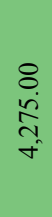 & 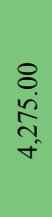 & 웅 & $\begin{array}{l}8 \\
0 \\
0\end{array}$ & $\begin{array}{l}8 \\
0 \\
0 \\
n \\
\infty \\
\infty\end{array}$ & $\begin{array}{l}8 \\
8 \\
8 \\
\text { ते } \\
\text { हิ }\end{array}$ & $\stackrel{8}{8}$ & $\begin{array}{l}8 \\
\dot{8} \\
8 \\
\text { m. } \\
\text { ஸे }\end{array}$ & $\begin{array}{l}8 \\
0 \\
0\end{array}$ & 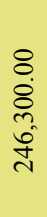 & 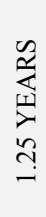 \\
\hline
\end{tabular}

Table 6a. Design, Manufacturing and Building Phases for the CASE study 1 and 2 - Working Days Efficiency (Author Source)

\begin{tabular}{|c|c|c|c|}
\hline $\mathbf{N r}$ & PROJECT EFFICIENCY LEVEL & COLOR INDICATION & DAYS \\
\hline 1 & HIGH EFFICIENCY & 0 & DAYS \\
\hline 2 & GOOD EFFICIENCY & 10 & DAYS \\
\hline 3 & MMEDIUMEFFICIENCY & 20 & DAYS \\
\hline 4 & LOW EFFICIENCY & 30 & DAYS \\
\hline 5 & VERY LOW EFFICIENCY & 40 & DAYS \\
\hline 6 & HAZARD SITUATION & 50 & DAYS \\
\hline
\end{tabular}


Table 6b. Design, Manufacturing and Building Phases for the CASE study 1 and 2 - Cost Efficiency (Author Source)

\begin{tabular}{|c|c|c|c|c|c|c|c|c|c|c|c|c|c|c|c|c|c|c|c|}
\hline 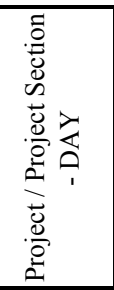 & 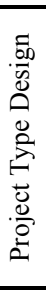 & 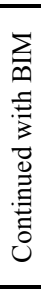 & 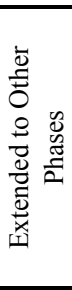 & 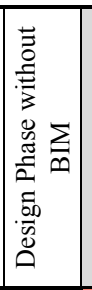 & 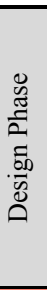 & 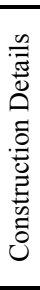 & $\begin{array}{l}0 \\
0 \\
0 \\
\frac{0}{0} \\
\overline{0} \\
\overline{0} \\
\frac{0}{0} \\
0 \\
0 \\
\bar{D} \\
\overline{0}\end{array}$ & 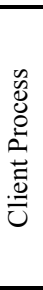 & 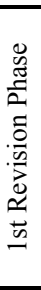 & 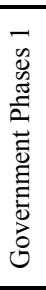 & 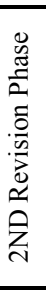 & 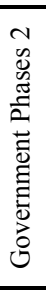 & 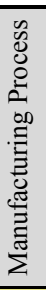 & 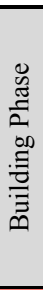 & 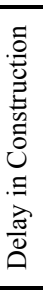 & 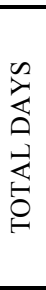 & 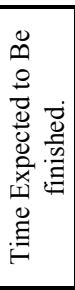 & 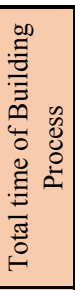 & 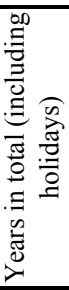 \\
\hline 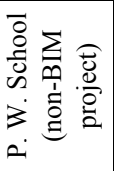 & 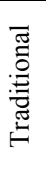 & & 蔦 & 0 & ஜे & $\infty$ & \& & 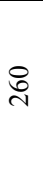 & లి & p & 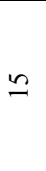 & $\cong$ & 8 & ঙิ & \& & $\begin{array}{l}\stackrel{\infty}{\infty} \\
\stackrel{\sim}{=}\end{array}$ & 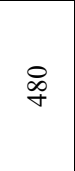 & $\begin{array}{l}\mathbb{8} \\
\infty\end{array}$ & 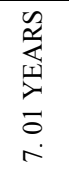 \\
\hline 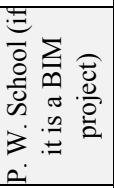 & $\sum_{m}$ & $\sum_{m}$ & $\sum_{m}$ & 0 & $\stackrel{\wp}{\curvearrowleft}$ & 0 & 0 & సి & 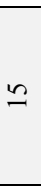 & ते & in & in & 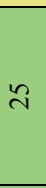 & \& & तి & $\stackrel{8}{\circ}$ & 0 & ஓ & 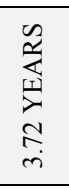 \\
\hline 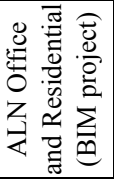 & 胥 & $\sum_{m}$ & $\sum_{\infty}$ & 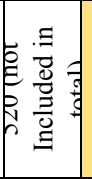 & ¿ & $\approx$ & 0 & 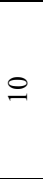 & 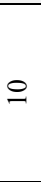 & 尺r & in & $\varrho$ & ஜ & 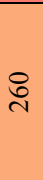 & 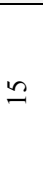 & $\stackrel{2}{\infty}$ & 0 & $\begin{array}{l}n \\
\infty \\
+\infty\end{array}$ & 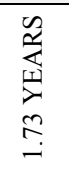 \\
\hline 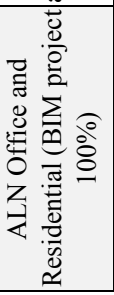 & $\sum_{m}$ & $\sum_{m}$ & $\sum_{m}$ & 0 & $\triangleright$ & 0 & 0 & 0 & in & in & 0 & 0 & $\varrho$ & $\underset{\sim}{\stackrel{+}{+}}$ & 0 & 守 & 0 & 悉 & 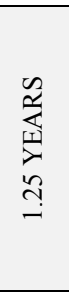 \\
\hline
\end{tabular}

\begin{tabular}{|c|c|c|c|c|c|c|c|c|c|c|c|c|c|c|c|c|c|c|c|}
\hline 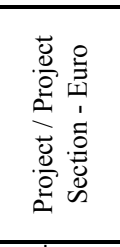 & 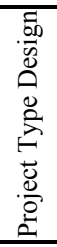 & 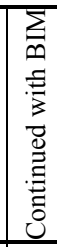 & 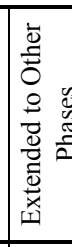 & 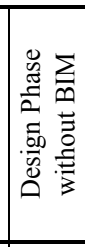 & 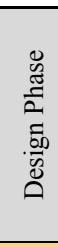 & 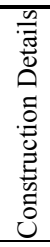 & 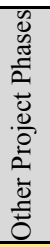 & 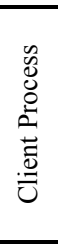 & 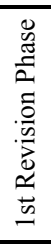 & 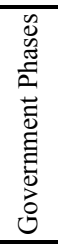 & 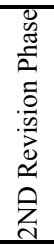 & 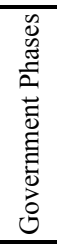 & 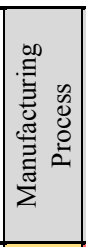 & 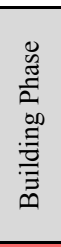 & 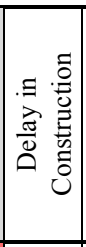 & 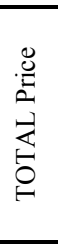 & 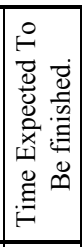 & 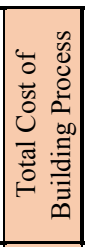 & 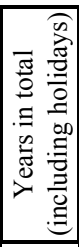 \\
\hline 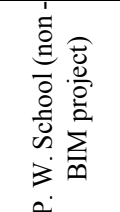 & 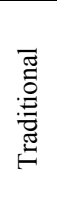 & 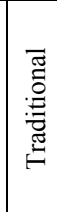 & 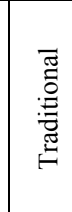 & 0 & $\begin{array}{l}8 \\
\dot{8} \\
\dot{8} \\
\dot{m}\end{array}$ & $\begin{array}{l}8 \\
8 \\
8 \\
8 \\
8 \\
8\end{array}$ & $\begin{array}{l}8 \\
8 \\
8 \\
8 \\
0\end{array}$ & $\begin{array}{l}8 \\
0 \\
0 \\
\infty \\
i \\
0 \\
0 \\
-1\end{array}$ & $\begin{array}{l}8 \\
8 \\
8 \\
n \\
\text { I } \\
=\end{array}$ & $\begin{array}{l}8 \\
\stackrel{0}{0} \\
\stackrel{0}{0}\end{array}$ & $\begin{array}{l}8 \\
\text {. } \\
\text { ते } \\
\text { in }\end{array}$ & $\begin{array}{c}8 \\
\dot{8} \\
8 \\
\text { of }\end{array}$ & 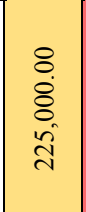 & $\begin{array}{l}8 \\
0 \\
8 \\
i \\
i \\
-1\end{array}$ & $\begin{array}{l}8 \\
\dot{9} \\
\dot{+} \\
\dot{0} \\
0 \\
\text { i }\end{array}$ & $\begin{array}{l}8 \\
0 \\
0 \\
o \\
0 \\
0 \\
n\end{array}$ & $\begin{array}{l}8 \\
8 \\
8 \\
8 \\
8 \\
8 \\
0 \\
-1\end{array}$ & $\begin{array}{l}8 \\
0 \\
0 \\
0 \\
\infty \\
0 \\
0 \\
0\end{array}$ & 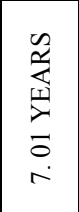 \\
\hline 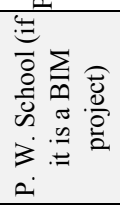 & $\sum_{m}$ & $\sum_{m}$ & $\sum_{m}$ & 0 & $\begin{array}{l}8 \\
\text { \&ें } \\
\text { n. } \\
\text { ปे }\end{array}$ & $\stackrel{8}{\circ}$ & $\stackrel{8}{0}$ & $\begin{array}{l}8 \\
0 \\
0 \\
0 \\
6 \\
6\end{array}$ & $\begin{array}{l}8 \\
\text {. } \\
\text { n. } \\
\text { त. }\end{array}$ & $\begin{array}{l}8 \\
\stackrel{0}{0} \\
\dot{0} \\
\dot{m}\end{array}$ & $\begin{array}{l}8 \\
\stackrel{0}{\circ} \\
\infty \\
\infty\end{array}$ & $\begin{array}{l}8 \\
\dot{8} \\
\stackrel{0}{0} \\
\sigma\end{array}$ & $\begin{array}{l}8 \\
: \\
\stackrel{5}{n} \\
\text { f }\end{array}$ & $\begin{array}{l}8 \\
\stackrel{8}{0} \\
\stackrel{8}{\circ}\end{array}$ & $\begin{array}{l}8 \\
8 \\
0 \\
\stackrel{0}{n} \\
\stackrel{0}{0}\end{array}$ & $\begin{array}{l}8 \\
\stackrel{0}{0} \\
0 \\
0 \\
\dot{J} \\
=\end{array}$ & $\stackrel{8}{\circ}$ & 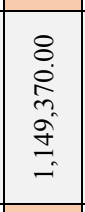 & 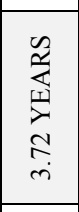 \\
\hline 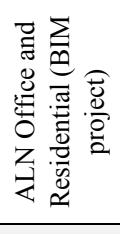 & 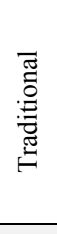 & $\sum_{\infty}$ & $\sum_{\infty}$ & 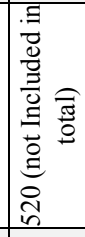 & $\begin{array}{l}8 \\
\dot{8} \\
\dot{8} \\
\dot{m}\end{array}$ & 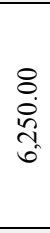 & $\stackrel{8}{8}$ & $\begin{array}{l}8 \\
0 \\
0 \\
n \\
\infty \\
\infty\end{array}$ & $\begin{array}{l}8 \\
\dot{8} \\
8 \\
0 \\
0\end{array}$ & $\begin{array}{l}8 \\
0 \\
6 \\
6 \\
\text { an }\end{array}$ & $\begin{array}{l}8 \\
\dot{8} \\
\dot{8} \\
\text { m. }\end{array}$ & $\begin{array}{l}8 \\
0 \\
0 \\
n \\
\infty \\
\infty\end{array}$ & 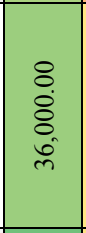 & $\begin{array}{l}8 \\
0 \\
8 \\
0 \\
0 \\
n\end{array}$ & 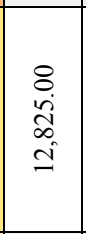 & $\begin{array}{l}8 \\
\text { i. } \\
\infty \\
i \\
i \\
\text { iे }\end{array}$ & $\stackrel{8}{\circ}$ & $\begin{array}{l}8 \\
\text { i. } \\
\infty \\
\text { ì } \\
\text { ते }\end{array}$ & 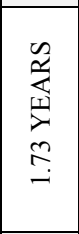 \\
\hline 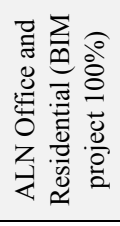 & 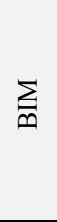 & $\sum_{m}$ & $\sum_{m}$ & 0 & $\begin{array}{l}8 \\
\dot{8} \\
8 \\
\dot{+}\end{array}$ & $\stackrel{8}{8}$ & $\stackrel{8}{0}$ & $\stackrel{8}{0}$ & $\begin{array}{l}\stackrel{i}{i} \\
\text { i } \\
\dot{v}\end{array}$ & 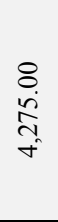 & $\stackrel{8}{0}$ & $\stackrel{8}{0}$ & $\begin{array}{l}8 \\
\stackrel{0}{0} \\
n \\
\infty \\
\infty\end{array}$ & 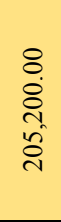 & $\stackrel{8}{0}$ & 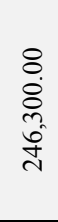 & $\stackrel{8}{\circ}$ & $\begin{array}{l}8 \\
\dot{8} \\
\text { n. } \\
\stackrel{+}{+} \\
\text { 1. }\end{array}$ & 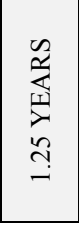 \\
\hline
\end{tabular}




\subsection{Design and Building Phases}

Based on the analysis, one clearly can see that the early stages of the design phase through the BIM project consume longer time in comparison to the traditional project. However, these early efforts can reduce significantly the timeline of the project development. Moreover, as can be seen in Table $6 \mathrm{~b}$, the total price of the project is significantly reduced within a BIM project compared to the traditional project. The hazard indications on red and orange relate to the manufacturing and building phase based on the traditional way of design. It shows that fewer efforts were made at the beginning and many problems occurred later in the manufacturing process and building phases.

The opposite occurred to a BIM project in which efforts are significantly high at the design phase, while in the manufacturing and building phases, the efforts are reduced to a minimal (Figure 1a, 1b). These results create a very reliable chart for a BIM project and rank the BIM project in a very safe and less risk on the management of the design phases and building phases.

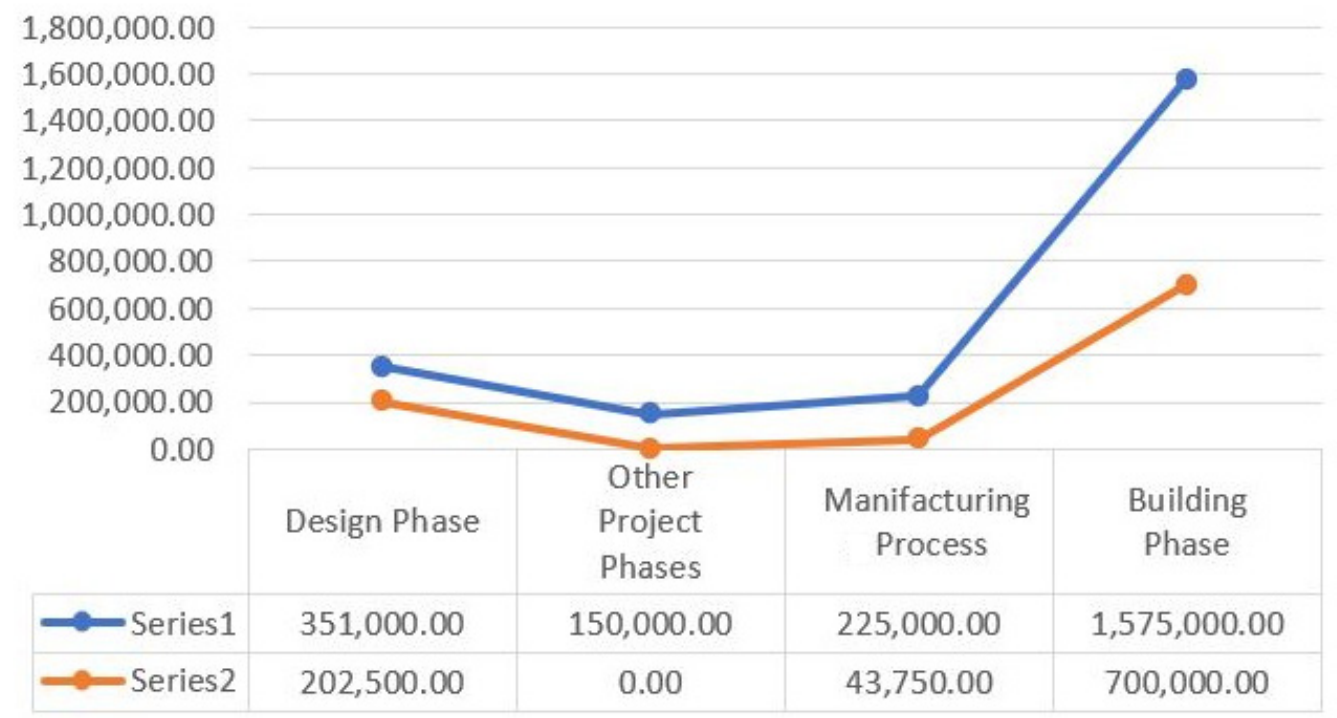

Figure 1a. Design, Manufacturing, and Building Phases for CASE study 1

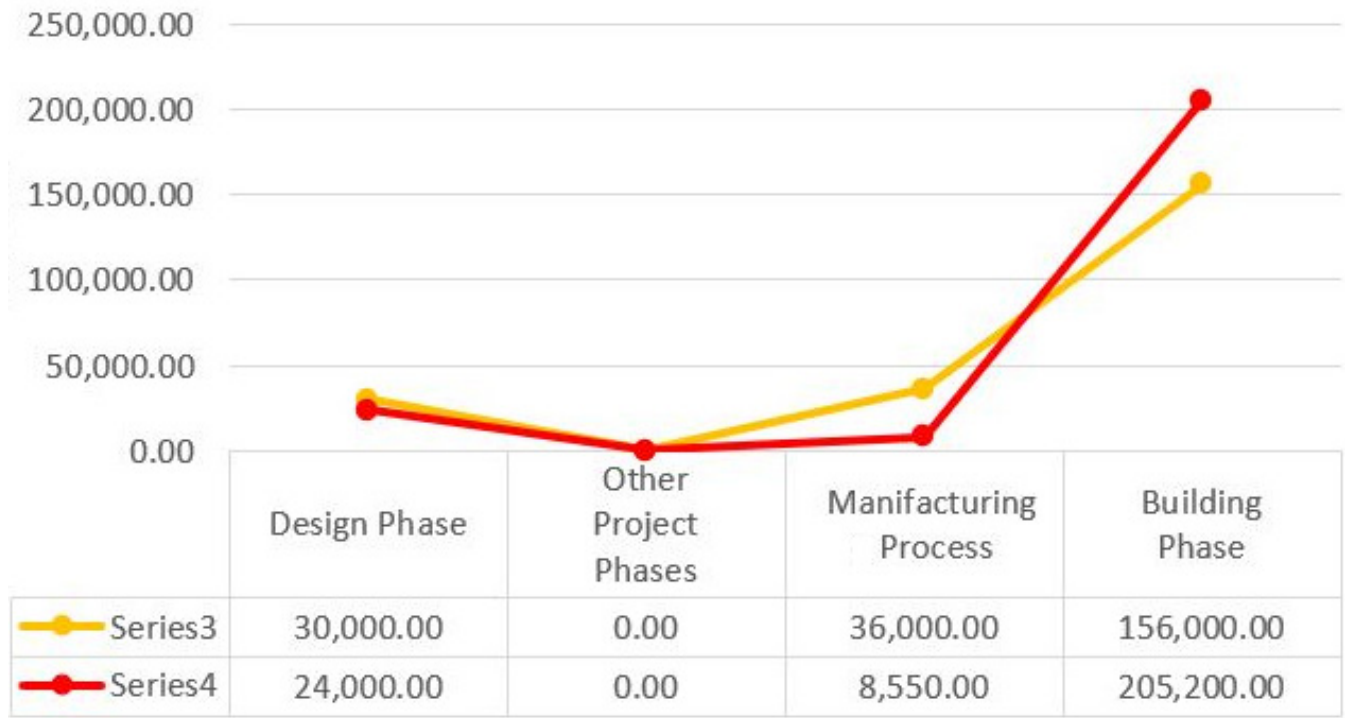

Figure 1b. Design, Manufacturing and Building Phases for CASE study 2 


\subsection{Delay and Revision Phases}

The second part of the research is focused on the delays that happen through traditional and BIM projects. These delays come mainly from managerial mistakes, less-skilled workers, no connectivity within stakeholders, etc.

The research analyzes found that those mistakes that cause delays on revisions of the design details, government revision, client revisions, client complaints, manufacturing delays, construction delays, etc, are the most difficult part to handle and consume a major part of the time and economic aspect of traditional management planning.

The case study 2, The BIM project, had a lot of delays because of revisions made manually by government authorities and controllers for project permission. Table 7a represents Working Days Efficiency for the Revision, Construction, Client, and Government delays phases for the CASE study 1 and 2. Table $7 \mathrm{~b}$ represents Cost Efficiency for the Revision, Construction, Client, and Government delays phases for the CASE study 1 and 2.

Table 7a. Revision, Construction, Client and Government delays for the CASE study 1 and 2 - Working Days Efficiency (Author Source)

\begin{tabular}{|c|c|c|c|c|c|c|c|c|c|c|c|c|c|c|c|c|c|c|c|}
\hline 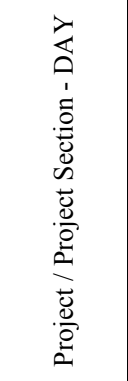 & 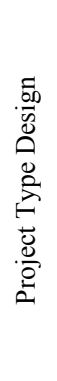 & 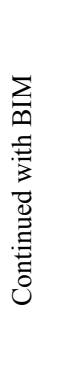 & 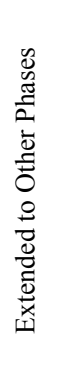 & 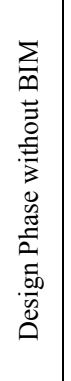 & 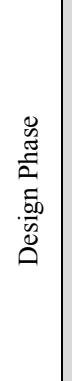 & 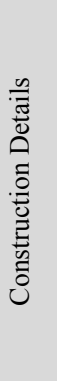 & 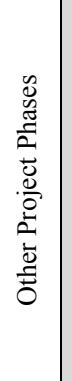 & 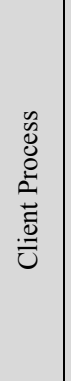 & 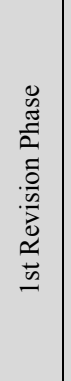 & 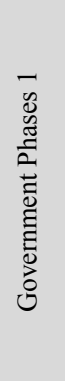 & 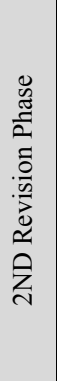 & 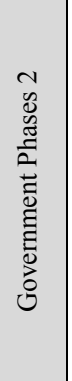 & 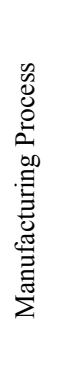 & 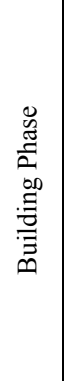 & 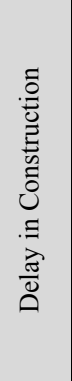 & 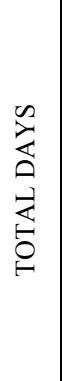 & 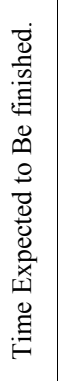 & 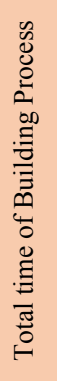 & 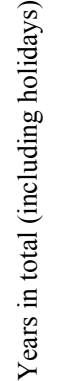 \\
\hline 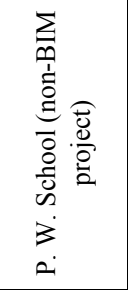 & 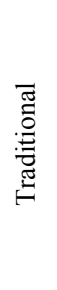 & 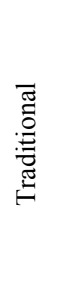 & 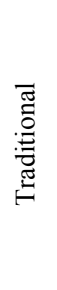 & 0 & ஓ్ల & $\triangleright$ & f & $\underset{N}{\mathbb{N}}$ & \&్ల & i & $\cong$ & $\stackrel{\sim}{2}$ & 8 & $\stackrel{\mathcal{\gamma}}{ }$ & q & $\begin{array}{l}\stackrel{\infty}{\infty} \\
\stackrel{2}{n}\end{array}$ & $\stackrel{\infty}{\stackrel{\infty}{+}}$ & $\begin{array}{l}\stackrel{8}{\infty} \\
\stackrel{\infty}{-}\end{array}$ & 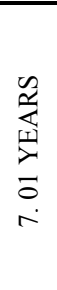 \\
\hline 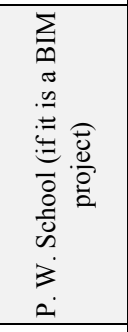 & $\sum_{m}$ & $\sum_{m}$ & $\sum_{m}$ & 0 & in & 0 & 0 & సి & 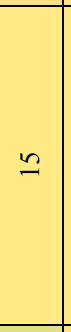 & त & in & in & $n$ & \& & సి & \& & 0 & ¿ & 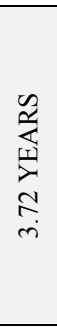 \\
\hline 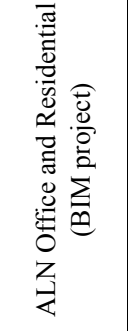 & 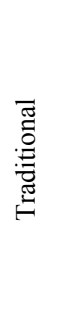 & $\sum_{m}$ & $\sum_{m}$ & 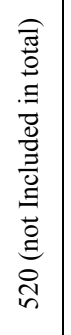 & 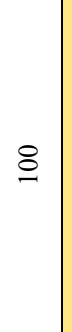 & $\ddot{v}$ & 0 & $\stackrel{\circ}{\circ}$ & $\stackrel{\circ}{\circ}$ & 凩 & in & $\stackrel{\circ}{\simeq}$ & $\stackrel{\sim}{ }$ & $\underset{\sim}{\stackrel{O}{0}}$ & 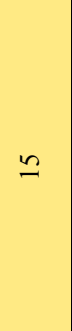 & $\stackrel{n}{\infty}$ & 0 & $\stackrel{n}{\infty}$ & 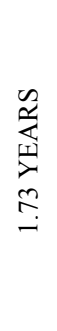 \\
\hline 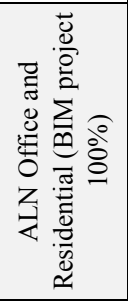 & $\sum_{m}$ & $\sum_{m}$ & $\sum_{\infty}$ & 0 & $\triangleright$ & 0 & 0 & 0 & in & in & 0 & 0 & $\stackrel{\circ}{ }$ & $\underset{d}{\stackrel{P}{N}}$ & 0 & 定 & 0 & $\stackrel{?}{\text { f }}$ & 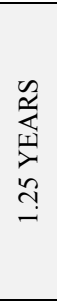 \\
\hline
\end{tabular}


Table 7b. Revision, Construction, Client and Government delays for the CASE study 1 and 2 - Cost Efficiency (Author Source)

\begin{tabular}{|c|c|c|c|c|c|c|c|c|c|c|c|c|c|c|c|c|c|c|c|}
\hline 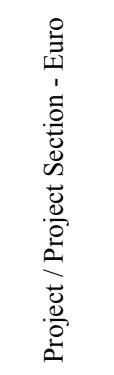 & 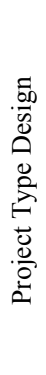 & 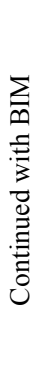 & 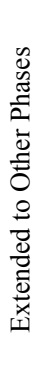 & 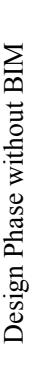 & 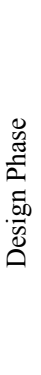 & 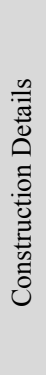 & 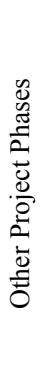 & 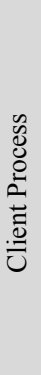 & 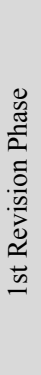 & 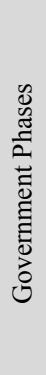 & 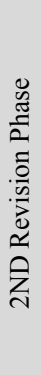 & 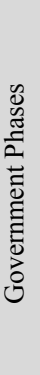 & 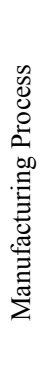 & 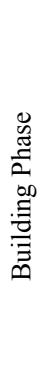 & 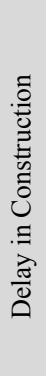 & 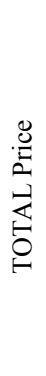 & 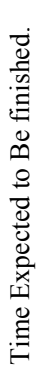 & 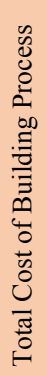 & 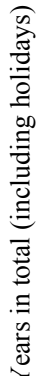 \\
\hline 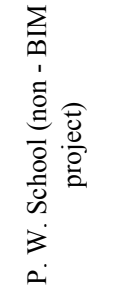 & 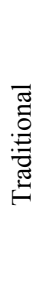 & 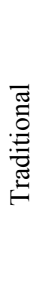 & 卺 & 0 & 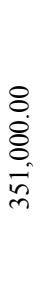 & $\begin{array}{l}8 \\
\dot{8} \\
8 \\
\dot{8} \\
\dot{8}\end{array}$ & 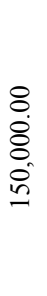 & 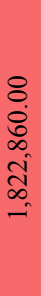 & 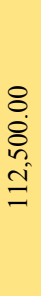 & $\begin{array}{l}8 \\
\dot{8} \\
8 \\
8\end{array}$ & 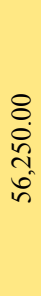 & $\begin{array}{l}8 \\
8 \\
8 \\
8 \\
\text { ஜ }\end{array}$ & 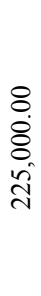 & $\begin{array}{l}8 \\
8 \\
8 \\
0 \\
n \\
i n \\
n\end{array}$ & 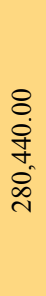 & $\begin{array}{l}8 \\
0 \\
0 \\
\infty \\
\infty \\
0 \\
n\end{array}$ & $\begin{array}{l}8 \\
\dot{8} \\
0 \\
8 \\
\dot{8} \\
-1\end{array}$ & $\begin{array}{l}8 \\
\circ \\
0 \\
\infty \\
\infty \\
0 \\
0\end{array}$ & 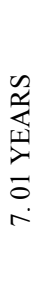 \\
\hline 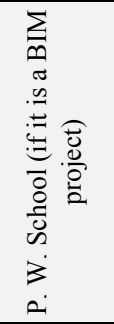 & $\sum_{m}$ & $\sum_{m}$ & 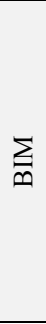 & 0 & 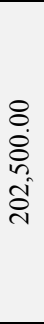 & $\stackrel{8}{8}$ & $\stackrel{8}{\circ}$ & $\begin{array}{l}8 \\
\dot{0} \\
\stackrel{0}{0} \\
\stackrel{6}{0}\end{array}$ & 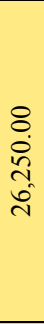 & $\begin{array}{l}8 \\
8 \\
8 \\
8 \\
\text { ஜ }\end{array}$ & $\begin{array}{l}8 \\
\stackrel{8}{\circ} \\
\infty \\
\infty\end{array}$ & $\begin{array}{l}8 \\
\dot{8} \\
\vdots \\
\circ\end{array}$ & $\begin{array}{l}8 \\
\dot{0} \\
\stackrel{n}{r} \\
\stackrel{f}{+}\end{array}$ & $\begin{array}{l}8 \\
\stackrel{8}{8} \\
8 \\
8 \\
8\end{array}$ & $\begin{array}{l}8 \\
8 \\
0 \\
\cdots \\
i\end{array}$ & $\begin{array}{l}8 \\
0 \\
0 \\
m \\
a \\
a \\
=\end{array}$ & $\stackrel{8}{\circ}$ & 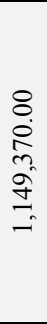 & 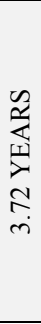 \\
\hline 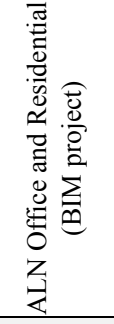 & 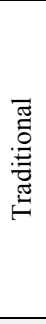 & $\sum_{m}$ & $\sum_{m}$ & 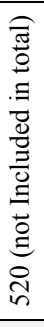 & 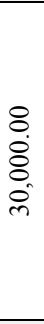 & 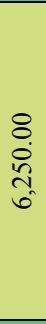 & $\stackrel{8}{\circ}$ & $\begin{array}{l}8 \\
\dot{8} \\
\tilde{n} \\
n \\
\infty\end{array}$ & $\begin{array}{l}8 \\
\dot{8} \\
8 \\
0 \\
0\end{array}$ & $\begin{array}{l}8 \\
0 \\
0 \\
0 \\
\tilde{n} \\
\end{array}$ & $\begin{array}{l}8 \\
8 \\
8 \\
\text { ஜ }\end{array}$ & 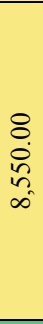 & $\begin{array}{l}8 \\
\dot{8} \\
8 \\
0 \\
0 \\
\text { D. }\end{array}$ & 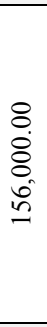 & $\begin{array}{l}8 \\
\ddot{1} \\
0 \\
\text { i } \\
\text { in }\end{array}$ & 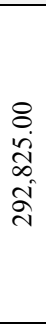 & $\stackrel{8}{\circ}$ & 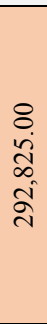 & 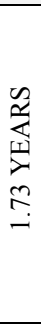 \\
\hline 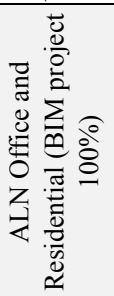 & 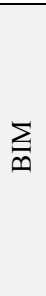 & $\sum_{m}$ & $\sum_{m}$ & 0 & $\begin{array}{l}8 \\
\stackrel{8}{8} \\
8 \\
\dot{0}\end{array}$ & $\stackrel{8}{8}$ & $\stackrel{8}{8}$ & : & 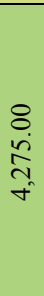 & 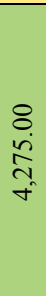 & $\stackrel{8}{\circ}$ & : & $\begin{array}{l}8 \\
0 \\
0 \\
n \\
\infty \\
\infty\end{array}$ & $\begin{array}{l}8 \\
\ddot{8} \\
\text { N } \\
\ddot{d} \\
\stackrel{i}{1}\end{array}$ & $\stackrel{8}{\circ}$ & 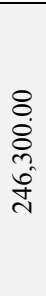 & $\stackrel{8}{\circ}$ & 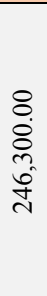 & $\begin{array}{l}\underset{1}{ \pm} \\
\underset{1}{2} \\
\stackrel{2}{n}\end{array}$ \\
\hline
\end{tabular}

Reading the values between traditional project and BIM project, one can visualize a significant difference between the two project plans. The traditional project has a very high level of delays at all phases, which makes the total schedule and economical aspects very perilous and puts the project at a higher risk of collapse (Table $7 \mathrm{a}$ and $7 \mathrm{~b}$ ). Different from the traditional method of design and construction, the percentage of the delays in BIM project can be lowered up to $15 \%$ from the total time of the project.

Reducing delays to the lowest level will provide a better possibility for the designers, clients, architects, manufacturers, and construction engineers to correct, recheck and enhance their plans, their schedules and create stronger bridges between all sections in a building chain (Figure 2a, 2b).

A lower number of revisions will be translated as a lower number of delays which in the economic aspect phase will save the project and the company a considerable amount $t$ of funds. 


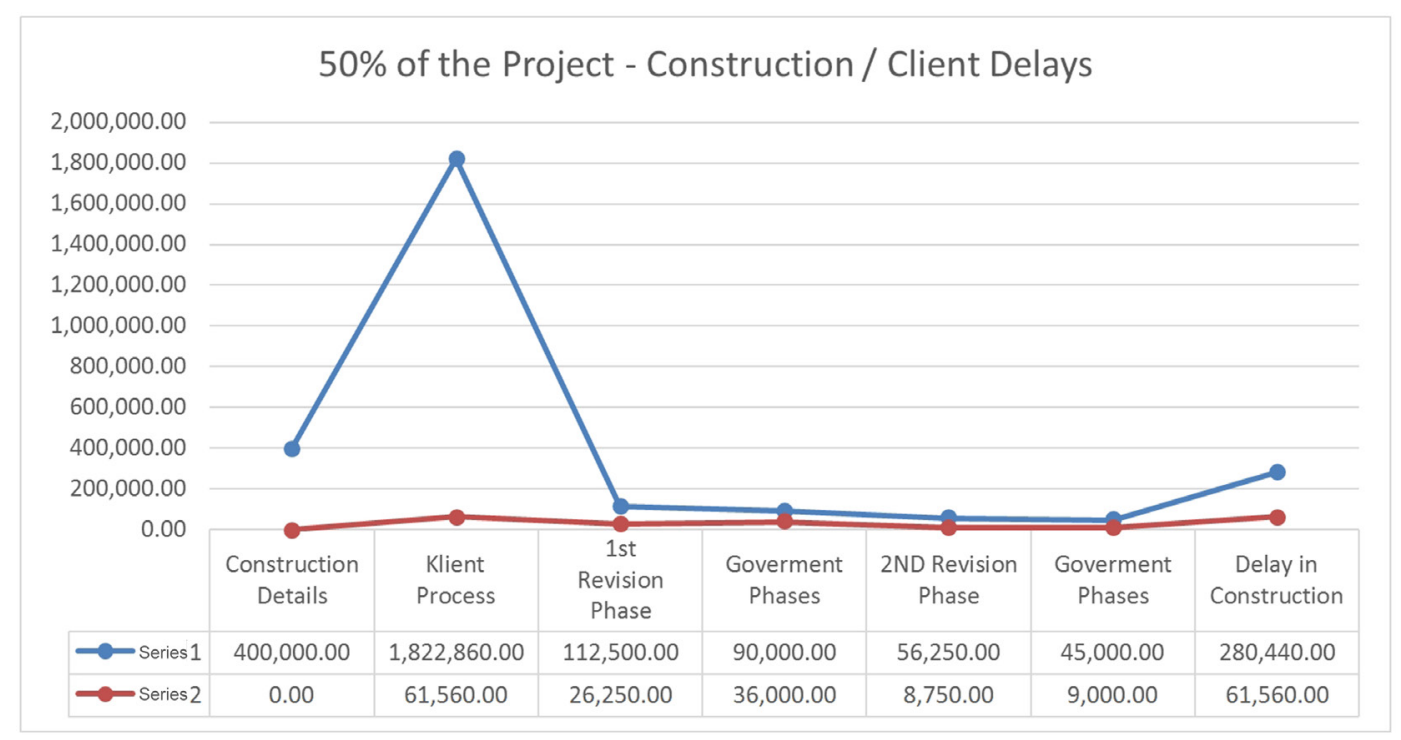

Figure 2a. Difference between Traditional Management and BIM

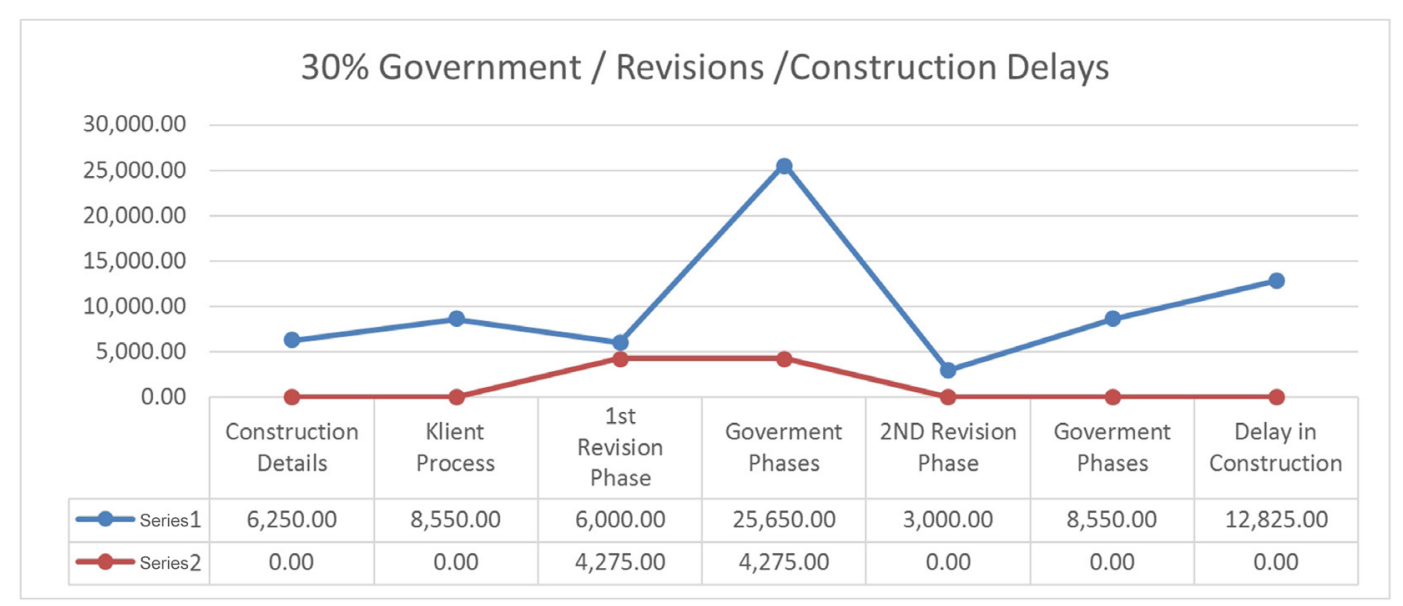

Figure 2b. Difference between Traditional Management and BIM

\subsection{Experts Involved}

Understanding project costs and delays through traditional project management or non-BIM projects, one of the significant costs that must be considered is related to the high number of experts involved in traditional projects (Figure 3 - Project Efficiency).

Based on the analysis, the BIM absent project had to include 41 team members. If the same project is realized on the BIM system, one would only need to allocate 18 team members.

The issue is not only related to the expenses over salaries, but also to the additional efforts required in planning and coordinating the tasks for all the experts.

In the table above, one can see that the delays in the traditional project cause additional costs for salaries and increase the total project costs for the architecture and engineering offices.

\subsection{Total Time of Building Process}

All the design problems, manufacturing, construction problems, mismanagement, miscommunication between stakeholders, unnecessary revisions, and other delays, obviously lead to the company facing a very unmanageable and problematic project (Fig. 4a, 4b). The analysis of the case studies show that there are many difficulties with the traditional project management which lead to an increase of the overall time of the construction process and also directly impact the finances of the project in terms of building assembly and building operations. 


\begin{tabular}{|r|r|r|r|}
\hline \multicolumn{2}{|c|}{} & NON - BIM \\
\hline $\mathrm{Nr}$ & Expert & Price Pre Expert $\mathrm{f}$ & Exp. Without BIM \\
\hline 1 & Architect Avarage Price / Day & 150 & 6 \\
\hline 2 & Enginiers Avarage Price / Per Day & 250 & 20 \\
\hline 3 & Other Experts Avarage Price / Per Day & 250 & 15 \\
\hline 4 & Municipality Costs & 300 & 10 \\
\hline 5 & Project Losts on Suspension & 171 & 41 \\
\hline
\end{tabular}

\begin{tabular}{|r|r|r|r|}
\hline $\mathrm{Nr}$ & Expert & Price Pre Expert f & Exp. With BIM \\
\hline 1 & Architect Avarage Price / Day & 150 & 3 \\
\hline 2 & Enginiers Avarage Price / Per Day & 250 & 8 \\
\hline 3 & Other Experts Avarage Price / Per Day & 250 & 7 \\
\hline 4 & Municipality Costs & 300 & 6 \\
\hline 5 & Project Losts on Suspension & 171 & 18 \\
\hline
\end{tabular}

Figure 3. Experts involved in the CASE study 1 without BIM and with BIM Management - Project Efficiency (Author Source)

\begin{tabular}{|c|c|c|c|c|}
\hline & TOTAL DAYS & $\begin{array}{r}\text { Time Expected To } \\
\text { Be finished. }\end{array}$ & $\begin{array}{r}\text { Total time of } \\
\text { Building Process }\end{array}$ & $\begin{array}{r}\text { Years in total } \\
\text { (including } \\
\text { holidays) }\end{array}$ \\
\hline Case 1 - TR & 1380 & 480 & 1860 & 7. 01 YEARS \\
\hline Case 1 - BIM & 960 & 0 & 960 & 3.72 YEARS \\
\hline Case 2- BIM & 485 & 0 & 485 & 1.73 YEARS \\
\hline ase $2-\operatorname{BIM}(100 \%) \longrightarrow$ & 340 & 0 & 340 & 1.25 YEARS \\
\hline
\end{tabular}

Figure 4a. Total time (working days) per CASE Study 1 (Author Source) 


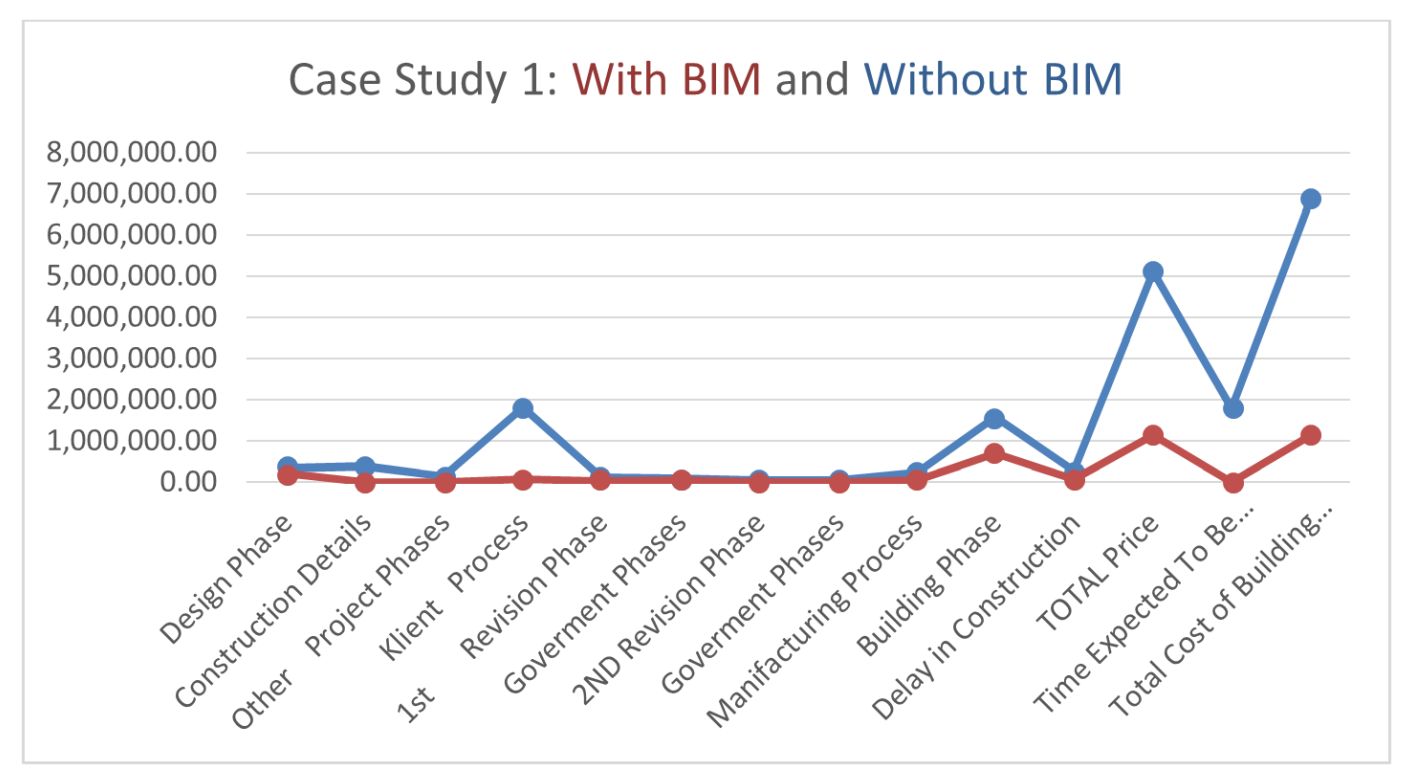

Figure 4b. Total time (working days) per Case Study 2 (Author Source)

\section{Summary and Implication}

The graphic chart as shown in figure 5 presents the workload comparison between case study 1 and case study 2. The amount of work that occurs during the design process for the BIM project of Case Study 2 is at a very high level, then it descends during the other phases of the process, and increases again during the construction phase, but it is still significantly low compared to the case study 1 (traditional design project). In this latter case, it can be seen that the efforts and the amount of work in the design phase are very low, while they increase significantly during the other phases. These efforts and workload culminate in the building phase. This increase in workload will lead to an increase in the number of errors, miscalculations, and poorly planned schedules. The extent of errors and calculations comes from overlapping of the works during the construction phase of the building. In this chart, we can see the difference between the level of BIM management and traditional management work. The efforts and the workload in the BIM management process take place very early in the design phase, which allows the entire process to be developed with low pressure in other phases. The opposite happens in traditional projects, where there is no single optimization plan between different phases, and there is an overlap between design and construction schedules and plans among different stakeholders.

One of the most important benefits of using BIM is saving money, reducing project cycle time, and eliminating construction schedule setbacks. In terms of design and information management, BIM allows design and documentation to be carried out simultaneously so that the documents can be easily changed to accommodate new information, and most importantly, delay times are controlled. This is clearly shown in the timeline of the project chart between Case Study 1 and Case Study 2. 


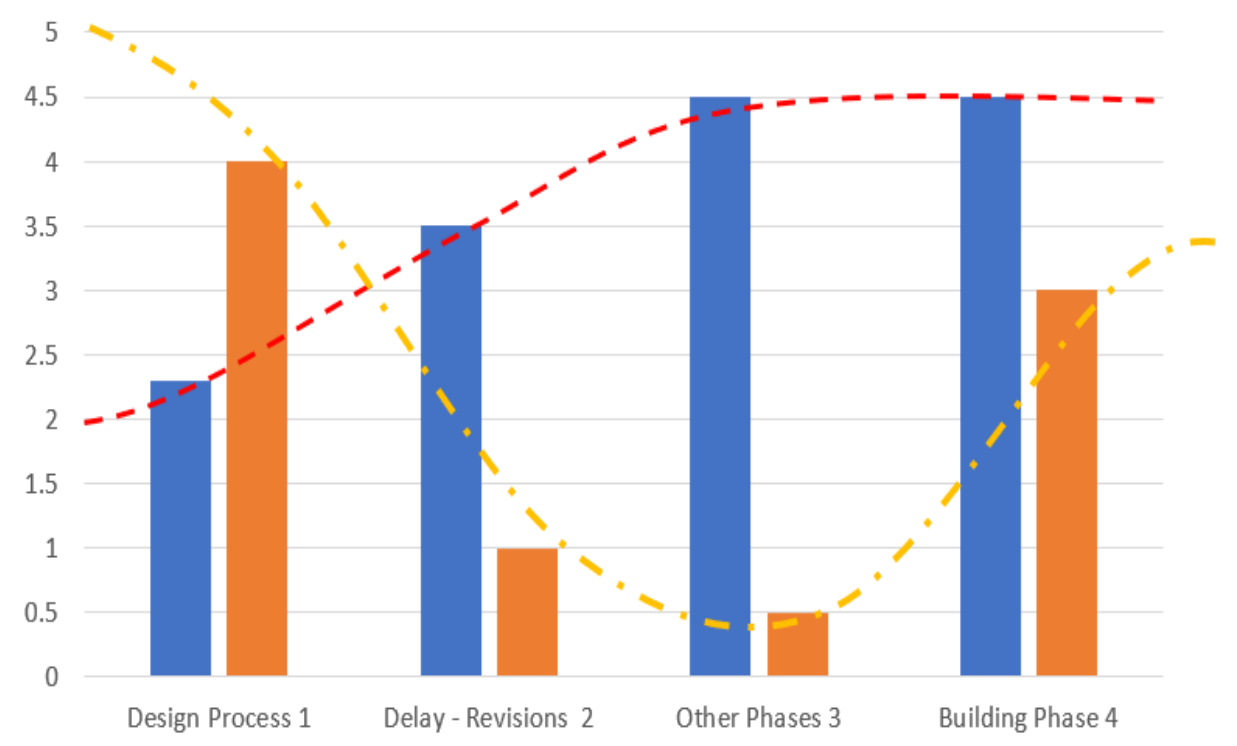

Figure 5. Difference between Case Study 1 (Blue) and Case Study 2 (Orange) (Author Source)

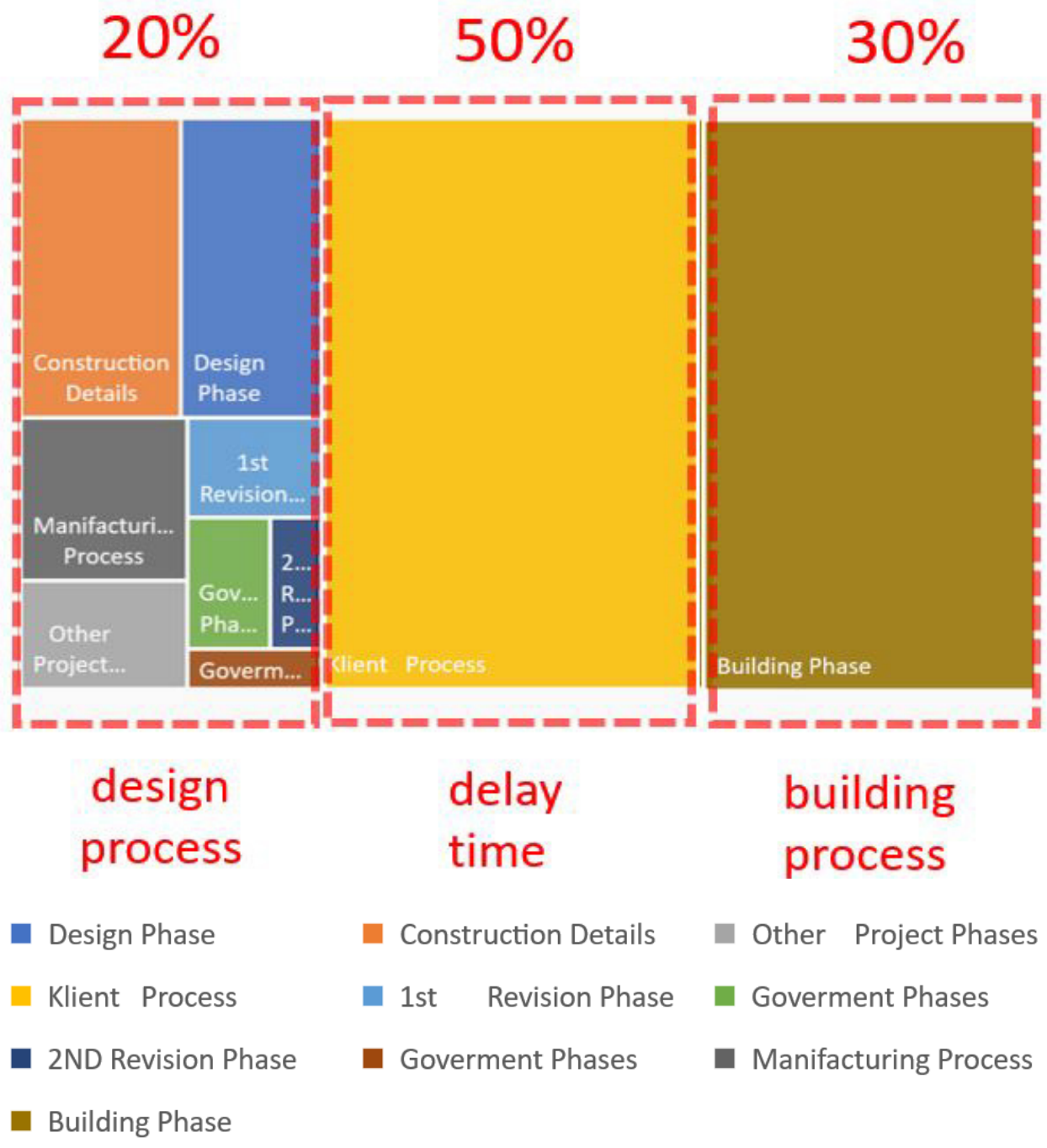

Figure 6a. Difference between Case Study 1 (Author Source) 


\section{$50 \% \quad 50 \%$}

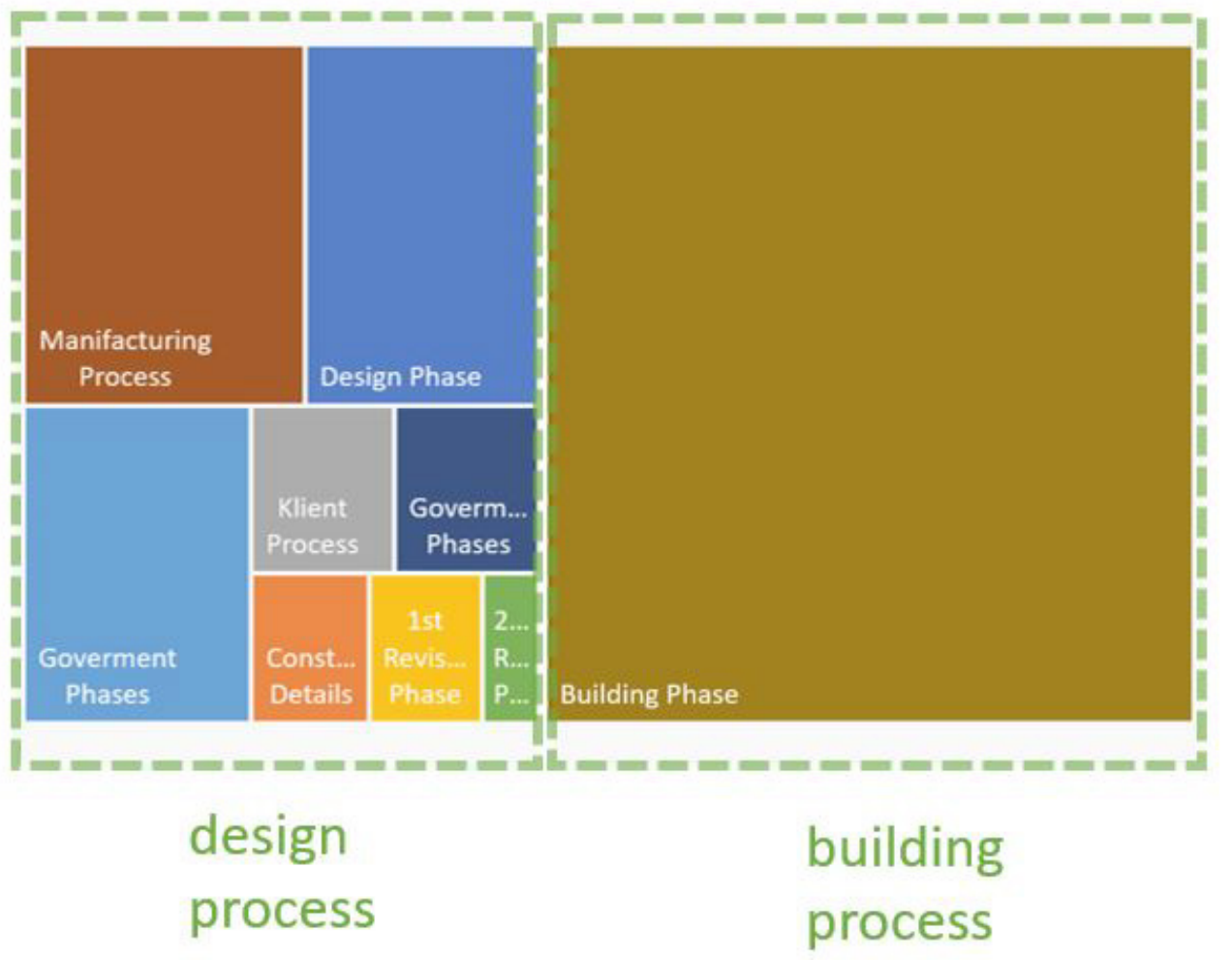

\section{Design Phase \\ Construction Details \\ Klient Process \\ 1st Revision Phase \\ Other Project Phases \\ 2ND Revision Phase \\ Goverment Phases \\ Goverment Phases \\ Manifacturing Process}

Building Phase

Figure 6b. Case Study 2 (Author Source)

In the first graph, Case Study 1 (Figure 6a) related to the overall project schedule, there is $20 \%$ design process, $50 \%$ delay time, and $30 \%$ build process. In case study 2 (Fig. 6b), with BIM management there is s $50 \%$ of the design process, and the other $50 \%$ was an explicit technical part of the construction process. The delay time is significantly reduced, only $5 \%$ in the design part. Project optimization is a very positive aspect of BIM management.

Having a better schedule within the BIM project allows for better planning, designing solutions, and enhancing management functions. In detail, this provides accurate planning, accurate communication and improved coordination. The BIM schedule ensures that the projects to be completed on time.

\section{Conclusions}

This research explores the use of the BIM management platform to reconstruct information management in the process of building design and construction.
Summarizing the differences between traditional and new management of design methods and new information management, after comparing the chronology of design phases, Tables and Figures between Case Study 1 - P.W School - Non-BIM Projects and Case Study 2 - ALN Office, Residential - Project using BIM, the timeline graphics in traditional format are noticeably slower in PW School project. The experience with BIM on the Case Study 2 - ALN Office, Residential Housing - Project suggests that information management can be improved and also that the design team management can be structured in a better way if one utilizes Building Information Modeling to connect all members within the same structure line.

The research will enhance and push forward the presence of BIM in different ranges in the German Design and Building Industry. It gives an opportunity to set criteria both in design, practical and managerial approaches, allowing access to the team participating and reviewing different phases of the project.

Although the research has its limitations since it's based 
on the German Design and Building Regulations - HOAI, this can be easily adapted to the other European countries, if we use the same parameters in the Eurocodes - Building Standards.

\section{REFERENCES}

[1] S. Musa, A. M. Ponting, F. A. A. Nifa, S. A. Shahron. Building Information Modeling (BIM) in Malaysian Construction Industry: Benefits and Future Challenges, AIP Conference Proceedings, 2018.

[2] M. Gouda, R. M. Abdallah, M. Marzoukc. BIM and Semantic Web-based Maintenance Information for Existing Buildings, Automation in Construction 116, 103209, 2020.

[3] M. H. Hanafi, G. G. Sing, S. Abdullah, R, Ismail. Organizational Readiness of Building Information Modelling Implementation: Architectural Practices, Jurnal Teknologi (Sciences \& Engineering), 2016.

[4] Ramaji, A. M. Memari. Product Architecture Model for Multistory Modular Buildings, J. Constr. Eng, Manage, 2016.

[5] Yu-Pin, M., Cheng, C., H. \& Mei-Chun, L. Combine BIM-Based and Mobile Technologies to Design On-Site Support System for the Communication and Management of Architectural Heritage Conservation Works, Proceedings of the IEEE International Conference on Applied System Innovation IEEE-ICASI - Meen, Prior \& Lam (Eds), 2017.

[6] Li, H., Lu, M., Hsu, S., C., M. Gray, Huang, T. Proactive Behavior-based Safety Management for Construction Safety Improvement, Safety Science, Vol. 75, 107-117, 2015.

[7] Li, J., D. Greenwood, M. Kassem. Blockchain in the Built Environment and Construction Industry: A Systematic Review, Conceptual Models and Practical use Cases, Automation in Construction, Vol. 102, 288-307, 2019.

[8] H. Abanda, C. Vidalakis, A. H. Oti, J. H. M. Tah. A Critical Analysis of Building Information Modelling Systems Used in Construction Projects, Advances in Engineering Software, Vol. 90, 183-201, 2015.

[9] Caetano, A. Leitão. Integration of an Algorithmic BIM Approach in a Traditional Architecture Studio, Journal of Computational Design and Engineering Vol. 6, 327-336, 2019.

[10] Lee, W., Kang, S., Moh, R., Wu, R., Hsieh, C. H., Shu, Z. Application of BIM Coordination Technology to HSR Changhua Station. Visualization in Engineering, Vol. 3, No. $1,2015$.

[11] M. R. Asl, S. Zarrinmehr, M. Bergin, W. Yan. BPOpt: A Framework for BIM-based Performance Optimization. Energy and Buildings Vol. 108, 401-412, 2015.

[12] Chen, K., Lu, W., Peng, Y., S. Rowlinson, Huang, Q. G. Bridging BIM and Building: From a Literature Review to an Integrated Conceptual Framework, International Journal of Project Management, Vol. 33, 1405-1416, 2015.

[13] Zhang, S., J. Teizer, N. Pradhananga, C. M. Eastman.
Workforce Location Tracking to Model, Visualize and Analyze Work Space Requirements in Building Information Models for Construction Safety planning, Automation in Construction, Vol. 60, 74-86, 2015.

[14] Zhang, S., K. Sulankivi, M. Kiviniemi, I. Romo, C. M. Eastman, J. Teizer. BIM-based Fall Hazard Identification and Prevention in Construction Safety Planning, Safety Science, Vol. 72, 31-45, 2015.

[15] D. Migilinskas, V. Popov, V. Juozevicius, L. Utinovichius. The Benefits, Obstacles and Problems of Practical BIM Implementation, Procedia Engineering, Vol. 57, 767-774, 2013.

[16] F. Banfi, K. Almarri. Management of the Organizational and Contractual Risks of BIM Projects in the Architecture, Engineering and Construction Industry (AEC). Faculty of Engineering \& IT, The British University, 2018.

[17] F. Banfi. Building Information Modelling - A Novel Parametric Modeling Approach Based on 3D Surveys of Historic Architecture Department of Architecture, Built Environment and Construction Engineering (ABC), 2013.

[18] J. Ramaji, J. I. Messner, E. Mostavi. IFC-based BIM to BEM Model Transformation, Online available from https://www.researchgate.net/publication/341025570 IFCBased_BIM-to-BEM_Model_Transformation [accessed Dec 12.2020], 2020.

[19] N. Kasim, N. A Zainal Abidin, R Zainal, N. Sarpin, M H I Abd Rahim and M. Saikah. Best practices of Building Information Modelling (BIM) Implementation in Design Phase for Construction Project. IOP Conf. Series: Materials Science and Engineering, 271, 012038, 2017.

[20] Ramaji, I. J., Messner, J. I. and Mostavi, E.. IFC-based BIM to BEM Model Transformation, 2020.

[21] L. Zheng, W. Lu Ke, Ch. Kwong Wing Chau, and Y. Niu.. Benefit sharing for BIM implementation: Tackling the moral hazard dilemma in inter-firm cooperation, 2017.

[22] Papadaki, I. N. and Chassiakos, A. P. Multi-objective Construction Site Layout Planning using Genetic Algorithms. Procedia Engineering 164, 20 - 27, 2016.

[23] H. Ashcraft, A. Khanzode, M. Fischer, D.Reed, Book: Integrating Project Delivery, 2017.

[24] Cheng, W. Chen, K. Chen, K. and Q. Wang, Study of Risk Evaluation for Complex Projects under BIM and IPD Collaborative Pattern Based on Neighborhood Rough Sets. Technical Gazette, 27, 2, 2019.

[25] Cheng, W. Chen, K. Chen, K. and Q. Wang, Data-driven Predictive Maintenance Planning Framework for MEP Components based on BIM and IoT using Machine Learning Algorithms. Automation in Construction, 112, 103087, 2020.

[26] R. Maskil-Leitan, U. Gurevich, I. Reychav, BIM Management Measure for an Effective Green Building Project, 2020.

[27] S. Viscuso C. Talamo A. Zanelli E. Arlati, BIM Management Guidelines of the Construction Process for General Contractors, 2020. 
[28] Darko, A. Chan, Y. Yang, M. O. Tetteh, Building information modeling (BIM)-based modular integrated construction risk management - Critical survey and future needs, 2020.

[29] Patacas, N. Dawood, M. Kassem, BIM for facilities management: A framework and a common data environment using open standards, 2020.

[30] P. Cortés-Pérez, A. Cortés-Pérez, P. Prieto-Muriel, BIM-integrated management of occupational hazards in building construction and maintenance, 2020.

[31] R. Pavón, A. A. Arcos Alvarez, M. Alberti, BIM-Based Educational and Facility Management of Large University Venues, 2020.

[32] Vigneault, C. Boton, H. Chong, B. Cooper-Cooke, An Innovative Framework of 5D BIM Solutions for
Construction Cost Management: A Systematic Review, 2020.

[33] C. D. De Gaetani, M. Mert, F. Migliaccio, Interoperability Analyses of BIM Platforms for Construction Management, 2020.

[34] R. Jang, W. H. Collinge, Improving BIM asset and facilities management processes: A Mechanical and Electrical (M\&E) contractor perspective, 2020.

[35] Zoghi, S. Kim, Dynamic Modeling for Life Cycle Cost Analysis of BIM-Based Construction Waste Management, 2020.

[36] B. Ali, H. Zahoor, A. R. Nasir, A. Maqsoom, R. Waqas A. Khan, K. Mateen Mazher, BIM-based claims management system: A centralized information repository for extension of time claims, 2020. 